\title{
Regular variation of fixed points of the smoothing transform
}

\author{
Xingang Liang a ${ }^{\mathrm{a}}$ and Quansheng Liu ${ }^{\mathrm{b}, \mathrm{c} *}$ \\ ${ }^{a}$ Beijing Technology and Business Univ., School of Science, 100048 Beijing, China \\ ${ }^{\text {b }}$ Univ. Bretagne-Sud, LMBA, UMR CNRS 6205, 56000 Vannes, France \\ ${ }^{c}$ Zhongnan University of Economics and Law, School of Statistics and Mathematics, 430073 Wuhan, China
}

Revised April 2019; accepted by Stoch. Proc. Appl. in November 2019

\begin{abstract}
Let $\left(N, A_{1}, A_{2}, \ldots\right)$ be a sequence of random variables with $N \in \mathbb{N} \cup\{\infty\}$ and $A_{i} \in \mathbb{R}_{+}$. We are interested in asymptotic properties of non-negative solutions of the distributional equation $Z \stackrel{\text { (d) }}{=} \sum_{i=1}^{N} A_{i} Z_{i}$, where $Z_{i}$ are non-negative random variables independent of each other and independent of $\left(N, A_{1}, A_{2}, \ldots\right)$, each having the same distribution as $Z$ which is unknown. For a solution $Z$ with finite mean, we prove that for a given $\alpha>1, \mathbb{P}(Z>x)$ is a function regularly varying at $\infty$ of index $-\alpha$ if and only if the same is true for $\mathbb{P}\left(Y_{1}>x\right)$, where $Y_{1}=\sum_{i=1}^{N} A_{i}$. The result completes the sufficient condition obtained by Iksanov \& Polotskiy (2006) on the branching random walk. A similar result on sufficient condition is also established for the case where $\alpha=1$.
\end{abstract}

AMS Subject Classification: 60K37, 60J80

Keywords: tail behavior, regular variation, smoothing transform, branching random walk, Mandelbrot's martingale

\section{Introduction and main results}

Let us introduce the smoothing transform that interests us. As usual, we write $\mathbb{N}=\{0,1, \ldots\}, \mathbb{N}^{*}=$ $\{1,2, \ldots\}, \overline{\mathbb{N}}=\mathbb{N} \cup\{\infty\}$ and $\mathbb{R}_{+}=[0, \infty)$. Let $\left(N, A_{1}, A_{2}, \ldots\right)$ be a random variable taking values in $\overline{\mathbb{N}} \times \mathbb{R}_{+} \times \mathbb{R}_{+} \times \cdots$. Assume that

$$
\mathbb{E} \sum_{i=1}^{N} A_{i}=1
$$

Let $\mathcal{M}_{1}$ be the set of probability measures on $\mathbb{R}_{+}$with finite mean. The smoothing transform $\mathcal{T}$ on $\mathcal{M}_{1}$ is defined by letting $\mathcal{T} \mu$ be the distribution of $\sum_{i=1}^{N} A_{i} Z_{i}$, where $Z_{i}$ are independent and identically distributed (i.i.d.) random variables with law $\mu$, independent of $\left(N, A_{1}, A_{2}, \ldots\right)$. It is convenient to write the fixed point equation $\mu=\mathcal{T} \mu$ in the equivalent form

$$
Z \stackrel{(\mathrm{d})}{=} \sum_{i=1}^{N} A_{i} Z_{i}
$$

where $Z_{i}$ are non-negative i.i.d. random variables independent of $\left(N, A_{1}, A_{2}, \ldots\right)$, each having the same law as $Z$ (which is unknown). By convention, the empty sum is taken to be 0 , so that $\sum_{i=1}^{N} A_{i}=\sum_{i=1}^{N} A_{i} Z_{i}=0$ if $N=0$. The smoothing transform $\mathcal{T}$ will be identified with the corresponding transform on Laplace transforms of elements of $\mathcal{M}_{1}$, so that by writing $\phi(s)=\mathbb{E} e^{-s Z}$, the equation (E) reads

$$
\phi(s)=(\mathcal{T} \phi)(s), \quad \text { where } \quad(\mathcal{T} \phi)(s)=\mathbb{E} \prod_{i=1}^{N} \phi\left(s A_{i}\right), \quad s \geq 0,
$$

with the convention that the empty product is taken to be 1 .

\footnotetext{
${ }^{*}$ Corresponding author

E-mail addresses: liangxingang@th.btbu.edu.cn (X. Liang), quansheng.liu@univ-ubs.fr (Q. Liu).
} 
The study of fixed points of the smoothing transform is interesting due to a large number of applications in a variety of applied probability settings, including branching processes, self-similar cascades, infinite particles systems, branching random walks, random fractals, the quicksort algorithm and the Pagerank algorithm (which is in the heart of the Google engine): see for example Bingham \& Doney (1974, 1975), Kahane \& Peyrière (1976), Biggins (1977), Durrett \& Liggett (1983), Rösler (1992), Liu (1998), Aldous \& Bandyopadhyay (2005), Biggins \& Kyprianou (2005), Iksanov \& Polotskiy (2006), Buraczewski (2009), Hu \& Shi (2009), Volkovich \& Litvak (2010), Barral \& Jin (2014), Buraczewski \& Kolesko (2014), Chen (2015) and Shi (2015).

A typical example for a solution of $(\mathrm{E})$ is the limit of a generalized Mandelbrot's martingale (see Liu (2000)) constructed as follows. Let $U=\bigcup_{n=0}^{\infty}\left(\mathbb{N}^{*}\right)^{n}$ be the set of all finite sequences, where $\left(\mathbb{N}^{*}\right)^{0}=\{\emptyset\}$ contains the null sequence $\emptyset$. Let $\left(N_{u}, A_{u 1}, A_{u 2}, \ldots\right)$, indexed by $u \in U$, be a sequence of independent copies of $\left(N, A_{1}, A_{2}, \ldots\right)$. Let $\mathbb{T}$ be the Galton-Watson tree with defining elements $\left\{N_{u}: u \in U\right\}$, that is: (i) $\emptyset \in \mathbb{T}$, (ii) $u i \in \mathbb{T}$ implies $u \in \mathbb{T}$, (iii) if $u \in \mathbb{T}$ and $i \in \mathbb{N}$, then $u i \in \mathbb{T}$ if and only if $1 \leq i \leq N_{u}$. Let $\mathbb{T}_{n}=\{u \in \mathbb{T}:|u|=n\}$ be the set of individuals in generation $n$, where $|u|$ denotes the length of the sequence $u$. Set

$$
Y_{n}=\sum_{u_{1} \ldots u_{n} \in \mathbb{T}_{n}} A_{u_{1}} \cdots A_{u_{1} \ldots u_{n}} \quad \text { for } n \geq 1
$$

Then $\left(Y_{n}\right)_{n \geq 1}$ is a non-negative martingale with respect to the filtration $\mathcal{E}_{n}=\sigma\left\{\left(N_{u}, A_{u 1}, A_{u 2}, \ldots\right):|u|<\right.$ $n\}, n \geq 1$, so that the limit

$$
Y_{\infty}=\lim _{n \rightarrow \infty} Y_{n}
$$

exists a.s. by the martingale convergence theorem, with $\mathbb{E} Y_{\infty} \leq 1$ by Fatou's lemma. By self-similarity of the construction, it can be easily seen that $Z=Y_{\infty}$ is a solution of (E). We shall see in Lemma 1.1 that any solution of (E) with finite mean can be obtained in this way.

Another typical example concerns the natural martingale occurring in the branching random walk (BRW) on the real line $\mathbb{R}$, described as follows. The initial particle $\emptyset$ of the 0 -th generation is located at $S_{\emptyset}=0 \in \mathbb{R}$; it gives birth to $N_{\emptyset}=N$ new particles $\emptyset i=i(1 \leq i \leq N)$ of the first generation with displacements $L_{\emptyset i}=L_{i}$. In general, each particle $u=u_{1} u_{2} \ldots u_{n}$ of $n$-th generation gives birth to $N_{u}$ new particles $u i\left(1 \leq i \leq N_{u}\right)$ of $(n+1)$-th generation, with displacements (relative to their parent) $L_{u i}$ on the real line, so that their positions are $S_{u i}=S_{u}+L_{u i}$. The random variables $\left(N_{u}, L_{u 1}, L_{u 2}, \ldots\right)$ are independent and identically distributed. Let $Z_{n}$ be the counting measure on the real line $\mathbb{R}$ which counts the number of particles of the $n$-th generation in a given set of $\mathbb{R}$ :

$$
Z_{n}(A)=\sum_{u \in \mathbb{T}_{n}} \mathbf{1}_{A}\left(S_{u}\right), \quad A \subset \mathbb{R}
$$

where $\mathbf{1}_{A}$ denotes the indicator function of $A$, and $\mathbb{T}_{n}$ is the set of individuals of generation $n$ as defined before. Let $t \in \mathbb{R}$ be fixed such that $m:=\mathbb{E} \sum_{i=1}^{N} e^{-t L_{i}}<\infty$. Then the Laplace transform of $Z_{n}$ at $t$ is $\tilde{Z}_{n}(t)=\int e^{-t x} d Z_{n}(x)=\sum_{u \in \mathbb{T}_{n}} e^{-t S_{u}}$, and the sequence

$$
W_{n}=\frac{\tilde{Z}_{n}(t)}{\mathbb{E} \tilde{Z}_{n}(t)}=\sum_{u \in \mathbb{T}_{n}} \frac{e^{-t S_{u}}}{m^{n}}
$$

(the normalized Laplace transform of $Z_{n}$ ) forms a non-negative martingale with respect to the filtration $\mathcal{E}_{n}=\sigma\left\{\left(N_{u}, L_{u 1}, L_{u 2}, \ldots\right):|u|<n\right\}, n \geq 1$. Notice that $\left\{W_{n}\right\}$ is just $\left\{Y_{n}\right\}$ with $A_{u}=e^{-t L_{u}} / m$, so that the a.s. limit variable $Z=\lim _{n} W_{n}$ is a solution of (E) with $A_{i}=e^{-t L_{i}} / \mathrm{m}$.

For $x \geq 0$, write

$$
\rho(x)=\mathbb{E} \sum_{i=1}^{N} A_{i}^{x} \quad \text { and } \quad \rho^{\prime}(x)=\mathbb{E} \sum_{i=1}^{N} A_{i}^{x} \ln A_{i}
$$

if the expectation exists with value in the extended real line $\overline{\mathbb{R}}=[-\infty, \infty]$. The following result concerns the existence and uniqueness of nontrivial solutions with finite mean, and the existence of moments of order $\alpha>1$. As usual, we write

$$
\ln ^{+} x=\max (\ln x, 0) \quad \text { and } \quad \ln ^{-} x=\max (-\ln x, 0) \quad \text { for } x \geq 0 .
$$


We always assume (1.1) and write $Y_{1}=\sum_{i=1}^{N} A_{i}$, in accordance with (1.2). Define for $x>0$,

$$
J(x):=\frac{x}{A(x)} \quad \text { with } \quad A(x)=\int_{0}^{x} \mathbb{E} \sum_{i=1}^{N} A_{i} \mathbf{1}_{\left\{-\ln A_{i}>t\right\}} d t=\mathbb{E} \sum_{i=1}^{N} A_{i} \min \left(\ln ^{-} A_{i}, x\right),
$$

where the last equality holds by Fubini's theorem. Let $J(0)=\lim _{x \downarrow 0} J(x)=1 / \mathbb{E} \sum_{i=1}^{N} A_{i} \mathbf{1}_{\left\{-\ln A_{i}>0\right\}}$. Notice that for any $x>0, A(x)=0$ if and only if a.s. $A_{i} \geq 1$ on $\left\{A_{i}>0\right\}$, for all $i=1, \cdots, N$. Consequently, if $\rho^{\prime}(1)<0$, then $A(1)>0$, and for any $x \geq 1, A(x) \geq A(1)>0$ and $J(x) \leq x / A(1)$. Notice also that $J(x)=1 / \int_{0}^{1} \mathbb{E} \sum_{i=1}^{N} A_{i} \mathbf{1}_{\left\{-\ln A_{i}>x u\right\}} d u$ is increasing in $x$.

Lemma 1.1 (i) Assume that $\rho^{\prime}(1)$ is well defined whose value may be infinite. Then the equation (E) has a solution $Z$ with $\mathbb{E} Z=1$ if and only if either

$$
\rho^{\prime}(1) \in(-\infty, 0) \text { and } \mathbb{E}\left[Y_{1} \ln ^{+} Y_{1}\right]<\infty,
$$

or

$$
\rho^{\prime}(1)=-\infty \quad \text { and } \quad \mathbb{E}\left[Y_{1} J\left(\ln ^{+} Y_{1}\right)\right]<\infty
$$

Moreover, if $Z$ is a solution of $(E)$ with $\mathbb{E} Z=1$, then $\mathbb{E} Y_{1}=1$ and $Z=Y_{\infty}$ in law, where $Y_{\infty}$ is defined by (1.3). (Consequently, there is only one solution $Z$ satisfying $\mathbb{E} Z=1$.)

(ii) Let $Z$ be a solution of (E) with $\mathbb{E} Z=1$. For $\alpha>1, \mathbb{E} Z^{\alpha}<\infty$ if and only if $\rho(\alpha)<1$ and $\mathbb{E} Y_{1}^{\alpha}<\infty$.

The first conclusion in Part (i) follows from a result of Alsmeyer and Iksanov (2009). In fact, by Theorem 1.3 of Alsmeyer and Iksanov (2009), when $\rho^{\prime}(1)$ is well defined, $\mathbb{E} Y_{\infty}=1$ (see (1.3) for the definition) if and only if (1.7) or (1.8) holds (actually their result is established in the context of branching random walks, but can also be formulated in the context of Mandelbrot's martingales). So to prove the conclusion we just need to show that the equation (E) has a solution $Z$ with $\mathbb{E} Z=1$ if and only if $\mathbb{E} Y_{\infty}=1$. The "if" part is evident. To see the "only if" part, it suffices to notice that by Theorem 7.1 of Liu (1998), if $Z$ is a solution of (E) with $\mathbb{E} Z=1$, then

$$
Y_{n} \rightarrow Z \quad \text { in law. }
$$

(To see this, it suffices to take $\eta(t)=e^{-t}$ and $\phi(t)=\mathbb{E} e^{-t Z}$ in Theorem 7.1 of Liu (1998); in this case, the condition (H1) therein is not needed). Hence $Z=Y_{\infty}$ in law. This argument also shows the second conclusion of Part (i).

Part (ii) is just Theorem 2.1 of Liu (2000) (although Liu only considered the case where $N<\infty$ a.s., this condition was not needed in the proof therein).

The criterion (1.7) has been well-known in the literature. For example, Biggins (1977) proved it under the additional condition $\mathbb{E} \sum_{i=1}^{\infty} A_{i}\left(\ln A_{i}\right)^{2}<\infty$, Liu (1997) proved it when $\mathbb{E} N<\infty$, Lyons (1997) proved it assuming only $\rho^{\prime}(1)$ is finite. In fact Biggins (1977) and Lyons (1997) also shown the sufficiency when $\rho^{\prime}(1)=-\infty$ and $\mathbb{E}\left[Y_{1} \ln ^{+} Y_{1}\right]<\infty$. Notice that, by the discussion before Lemma 1.1, if $\rho^{\prime}(1) \in[-\infty, 0)$, then $\mathbb{E}\left[Y_{1} \ln ^{+} Y_{1}\right]<\infty$ implies $\mathbb{E}\left[Y_{1} J\left(\ln ^{+} Y_{1}\right)\right]<\infty$.

A fundamental problem on the smoothing transform concerns the tail behavior of fixed points. Many authors have studied this problem in various contexts, see for example: Bingham \& Doney $(1974,1975)$ for Galton-Watson processes and Crump-Mode-Jirina processes; Kahane \& Peyrière (1976), Guivarc'h (1990), Barral (1999), Liu (2000), Barral \& Jin (2014), and Liang \& Liu (2015) for Mandelbrot's cascades; Durrett \& Liggett (1983) for some infinite particles systems; Biggins \& Kyprianou (1997,2005), Iksanov \& Polotskiy (2006) and Alsmeyer \& Kuhlbusch (2010) for branching random walks; Volkovich \& Litvak (2010) for the Pagerank algorithm (which is in the heart of the Google engine); Liu (1997, 1998), Aldous \& Bandyopadhyay (2005), Buraczewski(2009), Buraczewski \& Kolesko (2014) and Shi (2015) for related topics and many other references. Here we shall study the regular variation of the tail probability $\mathbb{P}(Z>x)$ when $x \rightarrow \infty$, for a solution $Z$ of (E) with finite mean.

Let $\alpha>1$ be such that $\rho(\alpha)<1$. Lemma 1.1 (ii) shows that $Z$ and $Y_{1}$ would have similar asymptotic properties. We shall show this by establishing comparison theorems between the tail behavior of $Z$ and that of $Y_{1}$. Throughout the paper, let

$$
R_{0}=\left\{\ell:[0, \infty) \rightarrow[0, \infty): \ell \text { is measurable and } \lim _{x \rightarrow \infty} \frac{\ell(\lambda x)}{\ell(x)}=1 \forall \lambda>0\right\}
$$


be the set of functions slowly varying at $\infty$ (also called the class of regularly varying functions of index 0 ). Recall that a function $\ell \in R_{0}$ can be represented in the form (cf. [11], p.12)

$$
\ell(x)=c(x) \exp \int_{a}^{x} \frac{\epsilon(u)}{u} d u \quad(x \geq a)
$$

for some $a>0$, where $c(\cdot)$ and $\epsilon(\cdot)$ are measurable with $c(x) \rightarrow c \in(0, \infty)$ and $\epsilon(x) \rightarrow 0$ as $x \rightarrow \infty$. Without loss of generality, we can take $c(x) \equiv c$. For simplicity, we take $c=1$. Also, as the value of $\ell(x)$ on finite intervals have no influence on our purpose, we can take $a=1$, and

$$
\ell(x)= \begin{cases}\exp \int_{1}^{x} \frac{\epsilon(u)}{u} d u & \text { if } x>1 \\ 1 & \text { if } x \leq 1\end{cases}
$$

where $\epsilon(\cdot)$ is measurable and bounded with $\epsilon(x) \rightarrow 0$ as $x \rightarrow \infty$.

As usual, we write $f(s)=o(g(s))$ if $f(s) / g(s) \rightarrow 0$, and $f(s) \sim g(s)$ if $f(s) / g(s) \rightarrow 1$, where $s \rightarrow 0$ or $\infty$ according to the context. We use

$$
f(s)=O(g(s)), \quad s \in I \quad(\text { resp. } s \rightarrow 0),
$$

to mean $|f(s)| \leq C|g(s)|$ on $I$ (resp. for $s>0$ small enough), where $C>0$ is a constant. We emphasize that $C>0$ is a deterministic constant when $g$ is a random function. For a set $B$ we use $\operatorname{Int} B$ to denote the interior of $B$.

In what follows we always assume that $Z \geq 0$ is a solution of $(\mathrm{E})$ with $\mathbb{E} Z=1$. Our main results are Theorems 1.1 and 1.2 below about the regular variation of $\mathbb{P}(Z>x)$.

Theorem 1.1 Let $\alpha>1$ be such that $\rho(a)<1$ and that

$$
\rho\left(\alpha+\delta_{0}\right)<\infty \quad \text { for some } \delta_{0}>0 .
$$

Then the following two assertions are equivalent for $\ell \in R_{0}$ :

$$
\begin{gathered}
\mathbb{P}\left\{Y_{1}>x\right\} \sim x^{-\alpha} \ell(x) \quad \text { as } x \rightarrow \infty, \\
\mathbb{P}\{Z>x\} \sim(1-\rho(\alpha))^{-1} x^{-\alpha} \ell(x) \quad \text { as } x \rightarrow \infty .
\end{gathered}
$$

In the context of the Galton-Watson process (where $A_{i}=1 / \mathbb{E} N<1$ for all $i$ ) and the Crump-ModeJirina process (where $A_{i} \leq 1$ for all $i$ ), the same result was established by Bingham \& Doney $(1974,1975)$ when $\alpha>1$ is not an integer, and by de Meyer (1982) when $\alpha>1$ is an integer. Their argument was based on the functional equation $\left(E^{\prime}\right)$ and a powerful Tauberian theorem on Laplace transforms. The implication $"(1.12) \Rightarrow(1.13) "$ has been extended by Iksanov \& Polotskiy (2006) to the general case where $A_{i}$ are not necessarily bounded, using an elegant martingale argument. The analytic argument of Bingham \& Doney (1975) depends heavily on the boundedness of $A_{i}$, while the probabilistic argument of Iksanov \& Polotskiy (2006) does not give the converse part. Notice that the boundedness condition on $A_{i}$ restricts much the applications: for example, in the typical case of the canonical Mandelbrot's cascade and in the branching Brownian motion, where $A_{i}$ are not bounded as $\ln A_{i}$ are Gaussian variables. Our theorem shows that the results of Bingham \& Doney $(1974,1975)$ and de Meyer $(1982)$ remain true in the general case (where $A_{i}$ are not necessarily bounded), so that they can be applied for example to general Mandelbrot's martingales and branching random walks; in our approach we extend the analytic arguments of Bingham \& Doney $(1974,1975)$ to the general case. While $A_{i}$ are not bounded, the arguments become much more delicate; we use delicate truncating technics to overcome the difficulty. Theorem 1.1 also improves the result of Iksanov \& Polotskiy (2006) in the sense that we obtain a necessary and sufficient condition, not just a sufficient condition.

When $\alpha \in(1,2)$, the implication " $(1.12) \Rightarrow(1.13)$ " has also been shown by Rösler, Topchii \& Vatutin $(2002,2003)$ for weighted branching processes with real weights and by Iksanov, Kolesko \& Meiners (2019) for a more general functional of the underlying branching random walk. Moreover, in the same work Iksanov et al. pointed out that when $\alpha \in(1,2)$ and when $\lim _{x \rightarrow \infty} \ell(x)$ exists and is strictly positive and finite, the condition (1.11) can be removed. For a similar situation, see Mikosch and Samorodnitsky (2000). In this paper we need the condition (1.11) to establish Theorem 2.1 about the relations between the reminders of the Taylor expansion of Laplace transforms of $Y_{1}$ and $Z$ (see e.g. the proof of Lemma 4.1 which serves 
for the proof of Theorem 2.1), and to secure a proper use of Potter's bound while using Theorem 2.1 to prove Theorem 1.1. We mention that we can avoid the use of Potter's bound (so that the condition (1.11) will not be used for this purpose), if $\ell$ is sub-multiplicative in the sense that $\ell(x y) \leq c \ell(x) \ell(y)$ for some constants $c, x_{0}>0$ and all $x, y \geq x_{0}$ (which holds in particular if $\lim _{x \rightarrow \infty} \ell(x) \in(0, \infty)$ ); however we still need (1.11) in the proof of Theorem 2.1, so that we fail to remove it in Theorem 1.1.

Closely related results on weighted moments of the form $\mathbb{E}\left(Z^{\alpha} \ell(Z)\right)$ have been established for example in Alsmeyer \& Kuhlbusch (2010) and Liang \& Liu (2015).

Remark 1.1 Notice that under (1.12) or (1.13), the condition (1.11) is equivalent to the condition

$$
\mathbb{E} A^{\alpha+\varepsilon}<\infty \quad \text { for some } \varepsilon>0, \quad \text { where } A=\sup _{1 \leq i \leq N} A_{i}
$$

which will be used in the following. In fact, if (1.11) holds, then it is clear that (1.14) holds for $\varepsilon=\delta_{0}$. So we only need to show that (1.14) implies (1.11). Relation (1.12) implies $\mathbb{E} Y_{1}^{\alpha-\delta_{0}}<\infty$ and (1.13) implies $\mathbb{E} Z^{\alpha-\delta_{0}}<\infty$ for $\delta_{0} \in(0, \alpha-1]$. So by Lemma 1.1(b), under (1.12) or (1.13), we have $\mathbb{E} Y_{1}^{\alpha-\delta_{0}}<\infty$. By Hölder's inequality,

$$
\rho\left(\alpha+\delta_{0}\right)=\mathbb{E} \sum_{i=1}^{N} A_{i}^{\alpha+\delta_{0}} \leq \mathbb{E}\left[Y_{1} A^{\alpha-1+\delta_{0}}\right] \leq\left(\mathbb{E} Y_{1}^{\alpha-\delta_{0}}\right)^{1 /\left(\alpha-\delta_{0}\right)} \cdot \mathbb{E}\left(A^{\alpha+\delta}\right)^{\left(\alpha-1-\delta_{0}\right) /\left(\alpha-\delta_{0}\right)}
$$

where $\delta=\frac{\left(\alpha+1-\delta_{0}\right) \delta_{0}}{\alpha-1-\delta_{0}}$. When $\delta_{0}>0$ is small enough, we have $\delta \leq \varepsilon$, so that the right side of $(1.15)$ is finite by $(1.14)$.

We shall prove the equivalence between (1.12) and (1.13) under the condition (1.14), using Tauberian theorems and truncating techniques. Our approach also gives a new proof of the result of Iksanov \& Polotskiy (2006).

Let $\alpha_{c} \geq 1$ be the critical value for the existence of moments of $Y_{1}$ :

$$
\alpha_{c}=\sup \left\{a \geq 1: \mathbb{E} Y_{1}^{a}<\infty\right\}
$$

Notice that Theorem 1.1 is applicable only in the case where $\alpha_{c}>1$. When $\alpha_{c}=1$, the following result shows that the situation is different. For $\ell \in R_{0}$ with $\int_{1}^{\infty} \ell(t) d t / t<\infty$, set $\tilde{\ell}(x)=0$ for $x \in[0,1)$, and

$$
\tilde{\ell}(x)=\int_{x}^{\infty} \frac{\ell(t)}{t} d t \quad \text { for } x \geq 1
$$

Theorem 1.2 Assume that $\rho\left(1+\delta_{0}\right)<\infty$ for some $\delta_{0}>0$, and that $\mu:=-\rho^{\prime}(1) \in(0, \infty)$. Let $\ell \in R_{0}$ be such that $\int_{1}^{\infty} \ell(x) d x / x<\infty$. If

$$
\mathbb{E} Y_{1} \mathbf{1}_{\left\{Y_{1}>x\right\}} \sim \ell(x) \quad(x \rightarrow \infty)
$$

then

$$
\mathbb{E} Z \mathbf{1}_{\{Z>x\}} \sim \mu^{-1} \tilde{\ell}(x) \quad(x \rightarrow \infty) .
$$

The same result was established by Bingham \& Doney $(1974,1975)$ for the Galton-Watson process, the Crump-Mode and Jirina processes. Notice that, just as in the result of Bingham \& Doney (1974, 1975), here we have not established the equivalence between (1.18) and (1.19). An explanation on this point will be given in Remark 7.1.

Theorems 1.1 and 1.2, without detailed proofs, have been announced in Liang \& Liu (2011). In both Theorems 1.1 and 1.2 , we are in the case where $\mathbb{E}\left[Y_{1} \ln ^{+} Y_{1}\right]<\infty$. In fact, in Theorem 1.1, each of the conditions (1.12) and (1.13) implies that $\mathbb{E}\left[Y_{1}^{a}\right]<\infty$ for $1<a<\alpha$, so that $\mathbb{E}\left[Y_{1} \ln ^{+} Y_{1}\right]<\infty$; in Theorem 1.2 , the conditions $\int_{1}^{\infty} \ell(x) d x / x<\infty$ and (1.18) also imply that $\mathbb{E}\left[Y_{1} \ln ^{+} Y_{1}\right]<\infty$, because, as mentioned in Bingham \& Doney (1974),

$$
\int_{1}^{\infty} \mathbb{E}\left[Y_{1} \mathbf{1}_{Y_{1}>x}\right] \frac{d x}{x}=\mathbb{E}\left[Y_{1} \ln ^{+} Y_{1}\right]
$$

As information, we mention that when $\mathbb{E}\left[Y_{1} \ln ^{+} Y_{1}\right]=\infty$, the asymptotic behavior of $Z$ has also been studied in the literature, see for example Alsmeyer \& Iksanov (2009) about the existence of moments of $Z$. 
The rest of the paper is organized as follows. In Section 2, we explain the main ideas of the approach, and the relations between the Laplace transforms of $Z$ and $Y_{1}$ (cf. Theorems 2.1 and 2.2). Section 3 contains some preliminary results about the equation (E) and its solutions. Sections 4 and 5 are devoted to the proof of auxiliary theorems announced in Section 2. Theorems 1.1 and 1.2 are proved respectively in Sections 6 and 7.

In the following sections, $C>0$ always stands for a deterministic constant whose value may differ from line to line.

\section{$2 \quad$ Key ideas of the approach}

\subsection{Tauberian theorems}

For a non-negative random variable $X$, we write $f(s)=\mathbb{E} e^{-s X}$ for its Laplace transform and $\mu_{r}=\mathbb{E} X^{r}$ for its $r$-moment $(r=0,1,2, \ldots)$. If $X$ has at least finite $n$-th moment, we set

$$
f_{n}(s)=(-1)^{n+1} \mathbb{E}\left\{e^{-s X}-\sum_{r=0}^{n} \frac{(-s X)^{r}}{r !}\right\}, \quad n \geq 0,
$$

and

$$
\begin{aligned}
f_{n}^{(n)}(s)=\frac{d^{n} f_{n}(s)}{d s^{n}} & =(-1)^{n}\left\{f^{(n)}(0)-f^{(n)}(s)\right\} \\
& =\mathbb{E} X^{n}\left(1-e^{-s X}\right), \quad n \geq 0
\end{aligned}
$$

We shall use the following two Tauberian theorems which give necessary and sufficient conditions for $\mathbb{P}(X>x) \sim x^{-\alpha} \ell(x)$, as $x \rightarrow \infty$ : see Proposition 2.1 for $\alpha \notin \mathbb{N}$, and Proposition 2.2 for $\alpha \in \mathbb{N}$. As usual, denote by $\Gamma(\cdot)$ the gamma function.

Proposition 2.1 ([9], Theorem $A)$ Let $X$ be a non-negative random variable with Laplace transform $f(s)=$ $\mathbb{E} e^{-s X}$. Let $n \in \mathbb{N}^{*}$. If $\mu_{r}=\mathbb{E} X^{r}<\infty$ for $r=0,1, \ldots, n$, then for $\alpha=n+\beta$ with $0 \leq \beta \leq 1$, the following statements are equivalent for $\ell \in R_{0}$ :

$$
\begin{array}{rlrl}
f_{n}(s) & \sim s^{\alpha} \ell(1 / s) & & (s \rightarrow 0) ; \\
f_{n}^{(n)}(s) & \sim \frac{\Gamma(\alpha+1)}{\Gamma(\beta+1)} s^{\beta} \ell(1 / s) & & (s \rightarrow 0) ; \\
\mathbb{E} X^{n} \mathbf{1}_{\{X>x\}} & \sim n ! \ell(x) & & \\
\mathbb{P}(X>x) & \sim \frac{(-1)^{n}}{\Gamma(1-\alpha)} x^{-\alpha} \ell(x) & (x \rightarrow \infty) & \text { when } \quad \beta=0 ; \\
\mathbb{E} X^{n+1} \mathbf{1}_{\{X \leq x\}} & \sim(n+1) ! \ell(x) & (x \rightarrow \infty) \quad \text { when } \quad 0<\beta=1 .
\end{array}
$$

Proposition 2.2 Le $X$ be a non-negative random variable with Laplace transform $f(s)=\mathbb{E} e^{-s X}$. Let $n \geq 0$ be an integer, and assume that $\mu_{n}=\mathbb{E} X^{n}<\infty$. Define $f_{n}$ by (2.1) and

$$
\hat{f}_{n}(s)=f_{n}(s) / s^{n}, \quad s>0 .
$$

Then the following two statements are equivalent for $\ell \in R_{0}$ :

$$
\begin{array}{rlrl}
\mathbb{P}(X>x) & \sim x^{-(n+1)} \ell(x) & & (x \rightarrow \infty) \\
\frac{\hat{f}_{n}(t)-\hat{f}_{n}(\lambda t)}{\ell(1 / t)} & \sim \frac{1}{n !} \ln \lambda \quad & (t \rightarrow 0) \quad \forall \lambda>1 .
\end{array}
$$

Proposition 2.1 was established by Bingham \& Doney (1974), using Karamata's theorem (cf. [11] or [19]). Proposition 2.2 can be obtained from the argument by de Meyer and Teugels (1980, Section 3) via de Haan's theorem (cf. [11], [15] or [16]), and was used in de Meyer (1982) for extending the result of Bingham and Doney (1974) to integer orders. However this result was not explicitly stated neither in de Meyer and Teugels (1980) nor in de Meyer (1982). For reader's convenience, we give the statement here whose proof is postponed in the Appendix. 


\subsection{Relations between the Laplace transforms of $Y_{1}$ and $Z$}

Assume that $Z \geq 0$ is a solution of $(\mathrm{E})$ with $\mathbb{E} Z=1$. In view of Propositions 2.1 and 2.2 , to obtain comparison results for the regular variations of $Y_{1}$ and $Z$, we first show relations between $h_{n}$ and $\phi_{n}$ defined below: for $n \in \mathbb{N}$,

$$
\begin{aligned}
& h_{n}(s)=(-1)^{n+1} \mathbb{E}\left\{e^{-s Y_{1}}-\sum_{r=0}^{n} \frac{\left(-s Y_{1}\right)^{r}}{r !}\right\}, \\
& \phi_{n}(s)=(-1)^{n+1} \mathbb{E}\left\{e^{-s Z}-\sum_{r=0}^{n} \frac{(-s Z)^{r}}{r !}\right\} .
\end{aligned}
$$

Let $\phi(s)=\mathbb{E} e^{-s Z}(s \geq 0)$ be the Laplace transform of $Z$. Set

$$
t(s)=-\ln \phi(s) \quad \text { and } \quad T(s)=\sum_{i=1}^{N} t\left(s A_{i}\right) .
$$

Since $\mathbb{E} Z=1$, by Jensen's inequality, we have $\phi(s)=\mathbb{E} e^{-s Z} \geq e^{-s \mathbb{E} Z}=e^{-s}$, so

$$
0 \leq t(s) \leq s \quad \text { and } \quad 0 \leq T(s) \leq Y_{1} s
$$

Moreover, as $\mathbb{E} Z=1$,

$$
t(s) \sim s \quad \text { and } \quad T(s) \sim \sum_{i=1}^{N} s A_{i}=s Y_{1} \quad(s \rightarrow 0) .
$$

For $n \in \mathbb{N}$, if $\mathbb{E} Z^{n}<\infty$, then $\phi$ has $n$-th continuous derivatives $\phi^{(n)}$ on $[0, \infty)$. Therefore $t(\cdot)$ and $T(\cdot)$ have also continuous $n$-th derivatives, denoted respectively by $t^{(n)}(s)$ and $T^{(n)}(s)$. Clearly, for $s \geq 0$,

$$
T^{(n)}(s)=\sum_{i=1}^{N} A_{i}^{n} t^{(n)}\left(s A_{i}\right)
$$

Let $M$ be a random variable whose distribution is determined by

$$
\mathbb{E} f(M)=\mathbb{E} \sum_{i=1}^{N} A_{i} f\left(A_{i}\right)
$$

for any measurable function $f:[0, \infty) \rightarrow[0, \infty)$. Set

$$
g_{n}(s)=\mathbb{E} Y_{1}^{n}\left(1-e^{-s Y_{1}}\right) \quad \text { and } \quad \psi_{n}(s)=\mathbb{E} Z^{n}\left(1-e^{-s Z}\right) .
$$

Then $g_{n}(s)=h_{n}^{(n)}(s)$ and $\psi_{n}(s)=\phi_{n}^{(n)}(s)$, where $h_{n}^{(n)}$ and $\phi_{n}^{(n)}$ are the $n$-th derivatives of $h_{n}$ and $\phi_{n}$, respectively. We have the following comparison theorem between $g_{n}$ and $\psi_{n}$.

Theorem 2.1 Let $Z \geq 0$ be a solution of $(E)$ with $\mathbb{E} Z=1$, and define $g_{n}$ and $\psi_{n}$ by (2.11).

(i) Assume that $\rho(\alpha)<1$ for some $\alpha>1$, and that $\rho\left(\alpha+\delta_{0}\right)<\infty$ for some $\delta_{0}>0$. Assume also that $\mathbb{E} Y_{1}^{\gamma}<\infty$ and $\mathbb{E} Z^{\gamma}<\infty$ for all $\gamma \in[1, \alpha)$. Then writing $\alpha=n+\beta$ with $n \in \mathbb{N}^{*}$ and $\beta \in(0,1]$, we have for $\epsilon>0$ small enough and all $s \geq 0$,

$$
\psi_{n}(s)-\mathbb{E}\left\{M^{n-1} \psi_{n}(s M)\right\}=g_{n}(s)+c_{n} s+O\left(s^{\beta+\epsilon}\right),
$$

where $c_{n}$ is a constant only depending on $n$, with $c_{n}=0$ when $\alpha$ is not an integer $($ i.e. $\beta<1)$.

(ii) Assume that $\rho\left(1+\delta_{0}\right)<\infty$ for some $\delta_{0}>0$. Then for $\epsilon>0$ small enough and all $s \geq 0$,

$$
\psi_{1}(s)-\mathbb{E} \psi_{1}(s M)=\mathbb{E}\left\{T^{\prime}(s)\left(1-e^{-T(s)}\right)\right\}+O\left(s^{\epsilon}\right) .
$$


Part (i) deals with the case where $\mathbb{E}\left(Y_{1}^{\gamma}\right)<\infty$ for some $\gamma>1$, while Part (ii) applies without any moment condition on $Y_{1}$ (other than $\mathbb{E} Y_{1}=1$ ).

To fix the idea, notice that the interesting part in (2.12) is the validity for $s \in[0,1]$ (or $s \rightarrow 0$ ), as the validity for $s \in(1, \infty)$ is evident due to the condition that $\mathbb{E} Y_{1}^{n}<\infty$ and $\mathbb{E} Z^{n}<\infty$ (which implies that each of the first three terms in (2.12) is bounded). The situation is similar for (2.13).

Integrating (2.12) and (2.13) $n$ times, and noting that $\psi_{k}(0)=g_{k}(0)=0$ for $k \leq n$, we obtain the following relations between $h_{n}$ and $\phi_{n}$.

Theorem 2.2 Let $Z \geq 0$ be a solution of $(E)$ with $\mathbb{E} Z=1$, and define $h_{n}$ and $\phi_{n}$ by (2.5) and (2.6).

(i) Assume that $\rho(\alpha)<1$ for some $\alpha>1$, and that $\rho\left(\alpha+\delta_{0}\right)<\infty$ for some $\delta_{0}>0$. Assume also that $\mathbb{E} Y_{1}^{\gamma}<\infty$ and $\mathbb{E} Z^{\gamma}<\infty$ for all $\gamma \in[1, \alpha)$. Then writing $\alpha=n+\beta$ with $n \in \mathbb{N}^{*}$ and $\beta \in(0,1]$, we have for $\epsilon>0$ small enough and all $s \geq 0$,

$$
\phi_{n}(s)-\mathbb{E}\left\{M^{-1} \phi_{n}(s M)\right\}=h_{n}(s)+c_{n} s^{n+1}+O\left(s^{\alpha+\epsilon}\right),
$$

where $c_{n}$ is a constant only depending on $n$, with $c_{n}=0$ when $\alpha$ is not an integer $($ i.e. $\beta<1)$.

(ii) Assume that $\rho\left(1+\delta_{0}\right)<\infty$ for some $\delta_{0}>0$. Then for $\epsilon>0$ small enough and all $s \geq 0$,

$$
\phi_{1}(s)-\mathbb{E}\left\{M^{-1} \phi_{1}(s M)\right\}=\mathbb{E}\left\{e^{-T(s)}-1+T(s)\right\}+O\left(s^{1+\epsilon}\right) .
$$

Just as in (2.12) and (2.13), what is essential in (2.14) and (2.15) is the validity for $s \in[0,1]($ or $s \rightarrow 0)$. Part (i) will be applied for the proof of Theorem 1.1, while Part (ii) applies for the proof of Theorem 1.2.

Let us explain one of the main difficulties in our approach compared with the work of Bingham and Doney (1975) who considered the case where $A_{i} \leq 1$. We just consider Part (ii) as an example for illustration. When $\sup _{1 \leq i \leq N} A_{i}$ is bounded, say $\sup _{1 \leq i \leq N} A_{i} \leq C$ a.s. for some constant $C>0$, then it can be easily seen that the function $\mathbb{E}\left(e^{-T(s)}-1+T(s)\right)$ appearing in Theorem 2.2 (ii) is close to $h_{1}(s)$ as $s \rightarrow 0$, which is essential for the proof of Theorem 1.2 (see Section 7). In fact, as $t(s) \sim s(s \rightarrow 0$ ), for each $\epsilon \in(0,1)$, there is $s_{0}=s_{0}(\epsilon)>0$ such that $(1-\epsilon) s \leq t(s) \leq(1+\epsilon) s, \forall s \in\left(0, s_{0}\right)$. As $A_{i} s \leq C s$ and $H(x):=e^{-x}-1+x$ is increasing, it follows that for $s \leq s_{0} / C, T(s)=\sum_{i=1}^{N} t\left(A_{i} s\right)$ lies between $(1 \pm \epsilon) s Y_{1}$, so that

$$
\mathbb{E}\left(e^{-T(s)}-1+T(s)\right) \text { lies between } \mathbb{E} H\left((1 \pm \epsilon) s Y_{1}\right)=h_{1}((1 \pm \epsilon) s) .
$$

When $\sup _{1 \leq i \leq N} A_{i}$ is not bounded, the argument above is no longer valid, so new ideas are needed to arrive at a similar conclusion: cf. Lemma 7.1; this is the typical difficulty that we have compared with the work of Bingham and Doney (1975).

We now summarize the key ideas for proving Theorem 1.1. In the case where $\alpha>1$ is not an integer, by Proposition 2.1, it suffices to show that the following two assertions are equivalent:

$$
\begin{array}{ll}
h_{n}(s) \sim s^{\alpha} \ell(1 / s) & (s \rightarrow 0), \\
\phi_{n}(s) \sim \frac{s^{\alpha} \ell(1 / s)}{1-\rho(\alpha)} & (s \rightarrow 0) .
\end{array}
$$

This equivalence will be shown by means of Theorem 2.2 (i). In the case where $\alpha>1$ is an integer, the argument is similar, using Proposition 2.2 instead of Proposition 2.1.

To prove Theorem 1.2, again by Proposition 2.1 (with $n=1$ and $\beta=0$ ), we just need to show that

$$
h_{1}(s) \sim s \ell(1 / s) \quad(s \rightarrow 0)
$$

implies

$$
\phi_{1}(s) \sim \mu^{-1} s \tilde{\ell}(1 / s) \quad(s \rightarrow 0),
$$

where $\tilde{\ell}$ is defined in (1.17). This will be done with help of Theorem 2.2 (ii). Unfortunately, we fail to prove the equivalence between (2.19) and (2.20), which would lead to the equivalence between (1.18) and (1.19). See Remark 7.1 on this point.

The details of the proofs of Theorems 1.1 and 1.2 are given respectively in Sections 6 and 7 . Both proofs are based on Theorem 2.2 about the comparison between $h_{n}$ and $\phi_{n}$, which is a direct consequence of Theorems 2.1 whose proof is given in Sections 4 and 5 . 


\section{Asymptotic properties of Laplace transforms}

Let $Z \geq 0$ be a random variable with $\mathbb{E} Z=1$. Set $\phi(s)=\mathbb{E} e^{-s Z}$ and $t(s)=-\ln \phi(s)$ for $s \geq 0$. In this section we present some asymptotic properties of $\phi(s), t(s)$ and their derivatives, as $s \rightarrow 0$.

As in Bingham and Doney (1975), differentiating $n$ times the function $\phi(s)$ in the form

$$
\phi(s)=e^{-t(s)},
$$

we obtain

$$
\phi^{(n)}(s)=e^{-t(s)}\left\{P_{n, t}(s)+\left(-t^{\prime}(s)\right)^{n}-t^{(n)}(s)\right\},
$$

where $P_{n, t}(s)$ is a polynomial in $t^{\prime}(s), \ldots, t^{(n-1)}(s)$ :

$$
P_{n, t}(s)=\sum_{a_{1}, \ldots, a_{n-1}} c\left(a_{1}, \ldots, a_{n-1}\right)\left(t^{\prime}(s)\right)^{a_{1}} \cdots\left(t^{(n-1)}(s)\right)^{a_{n-1}},
$$

$c\left(a_{1}, \ldots, a_{n-1}\right)$ are integers and $\left\{a_{k}\right\}_{k=1}^{n-1}$ are non-negative integers satisfying

$$
\sum_{k=1}^{n-1} k a_{k}=n \quad \text { and } \quad a_{1} \neq n
$$

by convention, $P_{1, t}(s)=P_{2, t}(s)=0$. By iteration, $t^{(n)}(s)$ can be written in the form

$$
t^{(n)}(s)=Q_{n, \phi}(s)+\left(\frac{\phi^{\prime}(s)}{\phi(s)}\right)^{n}+\left(-\frac{\phi^{(n)}(s)}{\phi(s)}\right),
$$

where $Q_{n, \phi}(s)$ is a polynomial in $\frac{\phi^{\prime}(s)}{\phi(s)}, \ldots, \frac{\phi^{(n-1)}(s)}{\phi(s)}$ :

$$
Q_{n, \phi}(s)=\sum_{a_{1}, \ldots, a_{n-1}} d\left(a_{1}, \ldots, a_{n-1}\right)\left(\frac{\phi^{\prime}(s)}{\phi(s)}\right)^{a_{1}} \ldots\left(\frac{\phi^{(n-1)}(s)}{\phi(s)}\right)^{a_{n-1}}
$$

$d\left(a_{1}, \ldots, a_{n-1}\right)$ are integers and $\left\{a_{k}\right\}_{k=1}^{n-1}$ are non-negative integers satisfying (3.4), with the convention that $Q_{1, \phi}(s)=Q_{2, \phi}(s)=0$.

The following elementary properties of $\frac{\phi^{(k)}(s)}{\phi(s)}$ and $t^{(k)}(s)$ will be frequently used.

Lemma 3.1 Let $Z \geq 0$ be a non-negative random variable. Write $\phi(s)=\mathbb{E} e^{-s Z}$ and $t(s)=-\ln \phi(s)$.

(i) If $\mathbb{E} Z^{n}<\infty$ for some $n \in \mathbb{N}^{*}$, then for $1 \leq k \leq n$,

$$
\sup _{s \geq 0}\left|\frac{\phi^{(k)}(s)}{\phi(s)}\right|<\infty, \quad \sup _{s \geq 0}\left|t^{(k)}(s)\right|<\infty,
$$

and for $0 \leq k \leq n-1$,

$$
\phi^{(k)}(s)=\phi^{(k)}(0)+O(s), \quad t^{(k)}(s)=t^{(k)}(0)+O(s), \quad s \geq 0 .
$$

(ii) If $\mathbb{E} Z^{n+\beta}<\infty$ for some $n \in \mathbb{N}^{*}$ and $\beta \in(0,1]$, then

$$
\phi^{(n)}(s)=\phi^{(n)}(0)+O\left(s^{\beta}\right) \quad \text { and } \quad t^{(n)}(s)=t^{(n)}(0)+O\left(s^{\beta}\right), \quad s \geq 0 .
$$

Consequently, the function $\phi_{n}$ defined by (2.6) satisfies

$$
\phi_{n}(s)=O\left(s^{n+\beta}\right), \quad s \geq 0 .
$$

We mention that what is important in (3.8)-(3.10) is the validity for $s \in[0,1]$ (or $s \rightarrow 0$ ).

Proof of Lemma 3.1. (i) Without loss of generality, we assume that $\mathbb{E} Z=1$. So the law $\mu$ of $Z$ satisfies $\mu([0,1])>0$. In order to prove (3.7), from the representation form (3.5), it is sufficient to show that $\frac{\phi^{(k)}(s)}{\phi(s)}$ 
are uniformly bounded on $[0, \infty)$ for $1 \leq k \leq n$. Recalling that $\phi$ is the Laplace transform of $Z$, we have for $1 \leq k \leq n$,

$$
\begin{aligned}
\left|\frac{\phi^{(k)}(s)}{\phi(s)}\right| & =\frac{\int_{[0, \infty)} t^{k} e^{-t s} \mu(d t)}{\int_{[0, \infty)} e^{-t s} \mu(d t)} \\
& \leq \frac{\int_{[0,1]} t^{k} e^{-t s} \mu(d t)}{\int_{[0,1]} e^{-t s} \mu(d t)}+\frac{\int_{(1, \infty)} t^{k} e^{-t s} \mu(d t)}{\int_{[0,1]} e^{-t s} \mu(d t)} \\
& \leq 1+\frac{e^{-s} \int_{(1, \infty)} t^{k} \mu(d t)}{e^{-s} \int_{[0,1]} \mu(d t)} \\
& \leq 1+\frac{\mathbb{E} Z^{k}}{\mu([0,1])},
\end{aligned}
$$

which is finite by the assumption. This ends the proof of (3.7). (3.8) is a consequence of (3.7) by the mean value theorem.

(ii) Notice that for $n \in \mathbb{N}$,

$$
\phi^{(n)}(s)-\phi^{(n)}(0)=(-1)^{n} \mathbb{E} Z^{n}\left(e^{-s Z}-1\right) .
$$

For any $\beta \in(0,1]$, we have $1-e^{-x} \leq C x^{\beta}(x>0)$, so

$$
1-e^{-s Z} \leq C s^{\beta} Z^{\beta} .
$$

It follows that for $n \in \mathbb{N}$ and $s \geq 0$,

$$
\left|\phi^{(n)}(s)-\phi^{(n)}(0)\right| \leq C s^{\beta} \mathbb{E} Z^{n+\beta} .
$$

This gives the first conclusion in (3.9). Using this inequality, together with (3.11), and the fact that $|\phi(s)-\phi(0)| \leq C s \leq C s^{\beta}$ for $s \in[0,1]$, we have

$$
\left|\frac{\phi^{(k)}(s)}{\phi(s)}-\frac{\phi^{(k)}(0)}{\phi(0)}\right| \leq\left|\frac{\phi^{(k)}(s)}{\phi(s)} \cdot \frac{\phi(0)-\phi(s)}{\phi(0))}\right|+\left|\frac{\phi^{(k)}(s)}{\phi(0)}-\frac{\phi^{(k)}(0)}{\phi(0)}\right| \leq C s^{\beta}, \quad s \in[0,1], \quad k=1, \ldots, n .
$$

It follows from the expression (3.5) of $t^{(n)}(s)$ that $\left|t^{(n)}(s)-t^{(n)}(0)\right| \leq C s^{\beta}$ for $s \in[0,1]$; the later holds evidently for $s>1$ since $\sup _{s \geq 0}\left|t^{(n)}(s)\right|<\infty$. This gives the second conclusion in (3.9). The proof of (3.9) is then finished.

Integrating (3.9) $n$ times, we see that (3.10) holds.

\section{Proof of Theorem 2.1: case where $\alpha \in[1, \infty) \backslash\{2,3, \ldots\}$}

Let $Z \geq 0$ be a solution of (E) with $\mathbb{E} Z=1$. We rewrite $\left(E^{\prime}\right)$ in the form

$$
\phi(s)=\mathbb{E} e^{-T(s)} .
$$

Differentiating $n$ times the equation ( $\left.\mathrm{E}^{\prime \prime}\right)$ just as we did for (3.1), we obtain

$$
\phi^{(n)}(s)=\mathbb{E} e^{-T(s)}\left\{P_{n, T}(s)+\left(-T^{\prime}(s)\right)^{n}-T^{(n)}(s)\right\},
$$

where $P_{n, T}(s)$ is defined as $P_{n, t}(s)$ (cf. (3.3)) with $t$ replaced by $T$.

Before giving the proof of Theorem 2.1, we first show two lemmas.

Lemma 4.1 If $\mathbb{E} Z^{n+\beta-\epsilon}<\infty$ and $\mathbb{E} \sum_{i=1}^{N} A_{i}^{n+\beta+\delta_{0}}<\infty$ for some $n \in \mathbb{N}^{*}, \beta \in(0,1], \delta_{0}>0$ and $\epsilon \in(0, \beta)$, then we have for $\epsilon>0$ small enough,

$$
0 \leq \mathbb{E} Y_{1}^{n}\left(e^{-T(s)}-e^{-s Y_{1}}\right)=O\left(s^{\beta+\epsilon}\right), \quad s \geq 0 .
$$


Proof. Notice that $t^{\prime}(0)=-\frac{\phi^{\prime}(0)}{\phi(0)}=1$ and that $\mathbb{E} Z^{1+\beta-\epsilon}<\infty$ implies $\left|t^{\prime}(s)-t^{\prime}(0)\right| \leq C s^{\beta-\epsilon}$ (by (3.9) of Lemma 3.1), so $|t(s)-s| \leq C \frac{s^{1+\beta-\epsilon}}{1+\beta-\epsilon}$. Hence

$$
0 \leq s Y_{1}-T(s) \leq C s^{1+\beta-\epsilon} A^{\beta-\epsilon} Y_{1},
$$

so

$$
\begin{aligned}
T(s) & \geq s Y_{1}-C s^{1+\beta-\epsilon} A^{\beta-\epsilon} Y_{1} \\
& =s Y_{1}\left(1-C s^{\beta-\epsilon} A^{\beta-\epsilon}\right) \\
& \geq s Y_{1} / 2 \quad \text { if } C s^{\beta-\epsilon} A^{\beta-\epsilon} \leq 1 / 2 .
\end{aligned}
$$

Now by the mean value theorem, $e^{-T(s)}-e^{-s Y_{1}}=e^{-\xi}\left(s Y_{1}-T(s)\right)$ for some $\xi \in\left[T(s), s Y_{1}\right]$, so that

$$
e^{-T(s)}-e^{-s Y_{1}} \leq e^{-T(s)}\left(s Y_{1}-T(s)\right) .
$$

Hence from (4.3) and (4.4), we see that if $C s^{\beta-\epsilon} A^{\beta-\epsilon} \leq 1 / 2$, then

$$
e^{-T(s)}-e^{-s Y_{1}} \leq e^{-s Y_{1} / 2}\left(s Y_{1}-T(s)\right) \leq C e^{-s Y_{1} / 2} s^{1+\beta-\epsilon} A^{\beta-\epsilon} Y_{1} .
$$

Using this and the fact that $e^{-x / 2} x^{1-2 \epsilon}$ is bounded for $x \geq 0$, we have

$$
\begin{aligned}
\left(e^{-T(s)}-e^{-s Y_{1}}\right) Y_{1}^{n} \mathbf{1}_{\left\{C s^{\beta-\epsilon} A^{\beta-\epsilon} \leq 1 / 2\right\}} & \leq C e^{-s Y_{1} / 2} s^{1+\beta-\epsilon} A^{\beta-\epsilon} Y_{1} \cdot Y_{1}^{n} \cdot 1 \\
& =C e^{-s Y_{1} / 2}\left(s Y_{1}\right)^{1-2 \epsilon} \cdot s^{\beta+\epsilon} A^{\beta-\epsilon} Y_{1}^{n+2 \epsilon} \\
& \leq C s^{\beta+\epsilon} A^{\beta-\epsilon} Y_{1}^{n+2 \epsilon} .
\end{aligned}
$$

On the other hand, as $\mathbf{1}_{\left\{C s^{\beta-\epsilon} A^{\beta-\epsilon}>1 / 2\right\}} \leq 2^{b} C^{b} s^{\beta+\epsilon} A^{\beta+\epsilon}$ with $b=(\beta+\epsilon) /(\beta-\epsilon)$, we have

$$
\begin{aligned}
\left(e^{-T(s)}-e^{-s Y_{1}}\right) Y_{1}^{n} \mathbf{1}_{\left\{C s^{\beta-\epsilon} A^{\beta-\epsilon}>1 / 2\right\}} & \leq Y_{1}^{n} \mathbf{1}_{\left\{C s^{\beta-\epsilon} A^{\beta-\epsilon}>1 / 2\right\}} \\
& \leq 2^{b} C^{b} s^{\beta+\epsilon} A^{\beta+\epsilon} Y_{1}^{n} .
\end{aligned}
$$

It follows from (4.5) and (4.6) that

$$
\left(e^{-T(s)}-e^{-s Y_{1}}\right) Y_{1}^{n} \leq C s^{\beta+\epsilon}\left(A^{\beta-\epsilon} Y_{1}^{n+2 \epsilon}+A^{\beta+\epsilon} Y_{1}^{n}\right) .
$$

Noting that $\mathbb{E} Z^{n+\beta-\epsilon}<\infty$ implies $\mathbb{E} Y_{1}^{n+\beta-\epsilon}<\infty$, by Hölder's inequality,

$$
\begin{aligned}
\mathbb{E} A^{\beta-\epsilon} Y_{1}^{n+2 \epsilon} & \leq\left(\mathbb{E} A^{(\beta-\epsilon) p}\right)^{1 / p} \cdot\left(\mathbb{E} Y_{1}^{(n+2 \epsilon) p^{*}}\right)^{1 / p^{*}}<\infty, \\
\mathbb{E} A^{\beta+\epsilon} Y_{1}^{n} & \leq\left(\mathbb{E} A^{(\beta+\epsilon) q}\right)^{\frac{1}{q}} \cdot\left(\mathbb{E} Y_{1}^{n q^{*}}\right)^{\frac{1}{q^{*}}}<\infty,
\end{aligned}
$$

where $p=\frac{n+\beta-\epsilon}{\beta-3 \epsilon}, p^{*}=\frac{n+\beta-\epsilon}{n+2 \epsilon}, q=\frac{n+\beta-\epsilon}{\beta-\epsilon}, q^{*}=\frac{n+\beta-\epsilon}{n}$ and $\epsilon>0$ is small enough such that $(\beta-\epsilon) p \leq$ $n+\beta+\delta_{0}$ and $(\beta+\epsilon) q \leq n+\beta+\delta_{0}$. Therefore (4.7) implies (4.2).

Further, when $n=1$, we also have the following lemma.

Lemma 4.2 If $\mathbb{E} Z^{1+\beta-\epsilon}<\infty$ and $\mathbb{E} \sum_{i=1}^{N} A_{i}^{1+\beta+\delta_{0}}<\infty$ for some $\beta \in(0,1], \delta_{0}>0$ and $\epsilon \in(0, \beta)$, then we have for $\epsilon>0$ small enough,

$$
\mathbb{E} T^{\prime}(s)\left(1-e^{-T(s)}\right)=g_{1}(s)+O\left(s^{\beta+\epsilon}\right), \quad s \geq 0 .
$$

Proof. Recall that $g_{1}(s)=\mathbb{E} Y_{1}\left(1-e^{-s Y_{1}}\right)$, so (4.10) is equivalent to

$$
\mathbb{E}\left(K_{1}+K_{2}\right)=O\left(s^{\beta+\epsilon}\right), \quad s \geq 0,
$$

where

$$
\begin{aligned}
& K_{1}=\left(T^{\prime}(s)-Y_{1}\right)\left(1-e^{-T(s)}\right), \\
& K_{2}=Y_{1}\left(e^{-s Y_{1}}-e^{-T(s)}\right) .
\end{aligned}
$$


In Lemma 4.1, we have shown that

$$
0 \geq \mathbb{E} K_{2} \geq C s^{\beta+\epsilon}, \quad s \geq 0,
$$

so we just need to show that

$$
0 \geq \mathbb{E} K_{1} \geq C s^{\beta+\epsilon}, \quad s \geq 0 .
$$

Notice that $K_{1} \leq 0$. In fact, Recalling the definition of $T^{\prime}(s)$, we have $T^{\prime}(s)-Y_{1}=\sum_{i=1}^{N} A_{i}\left[t^{\prime}\left(s A_{i}\right)-t^{\prime}(0)\right]$. Since $t^{\prime}(s)$ is non-increasing, it follows that $T^{\prime}(s)-Y_{1} \leq 0$, so that $K_{1} \leq 0$.

As $\mathbb{E} Z^{1+\beta-\epsilon}<\infty$, we have $\left|t^{\prime}(s)-t^{\prime}(0)\right| \leq C s^{\beta-\epsilon}$ for $s \geq 0$ (by (3.9) of Lemma 3.1), so that

$$
\left|T^{\prime}(s)-Y_{1}\right| \leq C s^{\beta-\epsilon} \sum_{i=1}^{N} A_{i}^{1+\beta-\epsilon} \leq C s^{\beta-\epsilon} A^{\beta-\epsilon} Y_{1} .
$$

Noting that $1-e^{-x} \leq x^{2 \epsilon}$ for $2 \epsilon \leq 1$ and $x>0$ and that $T(s) \leq s Y_{1}$, we obtain

$$
\begin{aligned}
\left|K_{1}\right| & \leq C s^{\beta-\epsilon} A^{\beta-\epsilon} Y_{1}(T(s))^{2 \epsilon} \\
& \leq C s^{\beta-\epsilon} A^{\beta-\epsilon} Y_{1}\left(s Y_{1}\right)^{2 \epsilon} \\
& =C s^{\beta+\epsilon} A^{\beta-\epsilon} Y_{1}^{1+2 \epsilon} .
\end{aligned}
$$

As $\mathbb{E} A^{\beta-\epsilon} Y_{1}^{1+2 \epsilon}<\infty$ (by (4.8)) for $\epsilon>0$ small enough, this implies (4.12). So the proof of (4.10) is finished.

Proof of Theorem 2.1: case where $\alpha \in[1, \infty) \backslash\{2,3, \ldots\}$. For $\alpha>1$ not an integer, write $\alpha=n+\beta$, where $n \in \mathbb{N}^{*}$ and $\beta \in(0,1)$. For $\alpha=1$, define $\beta=0$. We distinguish two cases according to $\alpha \in[1,2)$ or $\alpha>2$. In the following, $\epsilon$ always stands for a number in $(0,1-\beta)$ small enough.

(a) Case $\alpha \in[1,2)$. When $\alpha=1$ (so $\beta=0$ ), we suppose that $\rho\left(1+\delta_{0}\right)<\infty$ for some $\delta_{0}>0$, and we need to prove (2.13), that is,

$$
\psi_{1}(s)-\mathbb{E} \psi_{1}(s M)=\mathbb{E}\left\{T^{\prime}(s)\left(1-e^{-T(s)}\right)\right\}+O\left(s^{\epsilon}\right), \quad \text { where } \psi_{1}(s)=\phi^{\prime}(s)+1 ;
$$

when $\alpha \in(1,2)$ (so $\beta \in(0,1)$ ), we assume that $\rho(\alpha)<1, \rho\left(\alpha+\delta_{0}\right)<\infty$ for some $\delta_{0}>0$, and that $\mathbb{E} Y_{1}^{\gamma}<\infty$ and $\mathbb{E} Z^{\gamma}<\infty$ for all $\gamma \in[1, \alpha)$, and we need to prove (2.12) with $n=1$, that is

$$
\psi_{1}(s)-\mathbb{E}\left\{\psi_{1}(s M)\right\}=g_{1}(s)+O\left(s^{\beta+\epsilon}\right) .
$$

To prove (4.14) and (4.15), we only need to prove that

$$
\phi^{\prime}(s)-\mathbb{E} \phi^{\prime}(s M)=\mathbb{E} T^{\prime}(s)\left(1-e^{-T(s)}\right)+O\left(s^{\beta+\epsilon}\right), \quad s \geq 0 .
$$

In fact, since $\psi_{1}(s)=\phi^{\prime}(s)+1$, in (4.16) we can replace $\phi^{\prime}$ by $\psi_{1}$ to obtain

$$
\psi_{1}(s)-\mathbb{E} \psi_{1}(s M)=\mathbb{E} T^{\prime}(s)\left(1-e^{-T(s)}\right)+O\left(s^{\beta+\epsilon}\right) .
$$

When $\beta=0$, this gives (4.14). When $\beta \in(0,1)$, (4.17) together with Lemma 4.2 imply (4.15).

It remains to prove (4.16). Since $\phi^{\prime}(s)=\mathbb{E}\left\{-T^{\prime}(s) e^{-T(s)}\right\}$ (cf. $\left(E^{\prime}\right)$ ), (4.16) is equivalent to

$$
\mathbb{E} T^{\prime}(s)+\mathbb{E} \phi^{\prime}(s M)=O\left(s^{\beta+\epsilon}\right), \quad s \geq 0 .
$$

As $T^{\prime}(s)=\sum_{i=1}^{N} A_{i} t^{\prime}\left(s A_{i}\right)$ and $t^{\prime}(s)=-\frac{\phi^{\prime}(s)}{\phi(s)}$, by the definition of $M$ (cf. (2.10)), we obtain

$$
\begin{aligned}
\mathbb{E} T^{\prime}(s)+\mathbb{E} \phi^{\prime}(s M) & =\mathbb{E}\left\{\sum_{i=1}^{N} A_{i} t^{\prime}\left(s A_{i}\right)+\sum_{i=1}^{N} A_{i} \phi^{\prime}\left(s A_{i}\right)\right\} \\
& =\mathbb{E}\left\{\sum_{i=1}^{N} A_{i}\left(e^{t\left(s A_{i}\right)}-1\right)\left(-\phi^{\prime}\left(s A_{i}\right)\right)\right\} \\
& =\mathbb{E}\left\{\sum_{i=1}^{N} A_{i}\left(1-\phi\left(s A_{i}\right)\right) \cdot\left[\frac{-\phi^{\prime}\left(s A_{i}\right)}{\phi\left(s A_{i}\right)}\right]\right\} .
\end{aligned}
$$


Since $\frac{1-\phi(x)}{x} \rightarrow 1(x \rightarrow 0)$ and $0<\beta+\epsilon \leq 1$, we have

$$
1-\phi(x) \leq C x^{\beta+\epsilon}, \quad x>0
$$

(the inequality is evident for $x>1$; if $x \leq 1$, then $1-\phi(x) \leq C x \leq C x^{\beta+\epsilon}$ ). So with the fact that $\sup _{s \geq 0}\left|\frac{-\phi^{\prime}(s)}{\phi(s)}\right|<\infty$ (by Lemma 3.1(i)) and $\mathbb{E} Z<\infty$, we have

$$
\begin{aligned}
\left|\mathbb{E} T^{\prime}(s)+\mathbb{E} \phi^{\prime}(s M)\right| & \leq C s^{\beta+\epsilon} \mathbb{E} \sum_{i=1}^{N} A_{i}^{1+\beta+\epsilon} \\
& =C s^{\beta+\epsilon} \rho(\alpha+\epsilon) .
\end{aligned}
$$

This gives (4.18) and therefore (4.16).

(b) Case $\alpha>2$ not an integer. So $\alpha=n+\beta$ with $n \in\{2,3, \ldots\}$ and $\beta \in(0,1)$. We assume the conditions of Theorem 2.1 with the given $\alpha$, and we need to prove (2.12). Notice that

$$
\psi_{n}(s)=(-1)^{n+1}\left[\phi^{(n)}(s)-\phi^{(n)}(0)\right] \quad \text { and } \quad g_{n}(s)=-\mathbb{E}\left[Y_{1}^{n}\left(e^{-s Y_{1}}-1\right)\right] .
$$

So (2.12) is equivalent to

$$
\left[\phi^{(n)}(s)-\phi^{(n)}(0)\right]-\mathbb{E} M^{n-1}\left[\phi^{(n)}(s M)-\phi^{(n)}(0)\right]=\mathbb{E}\left[\left(-Y_{1}\right)^{n}\left(e^{-s Y_{1}}-1\right)\right]+O\left(s^{\beta+\epsilon}\right) .
$$

To prove (4.20), we only need to show that for all $s \geq 0$,

$$
\phi^{(n)}(s)-\mathbb{E} M^{n-1} \phi^{(n)}(s M)=\mathbb{E} e^{-s Y_{1}}\left(-Y_{1}\right)^{n}+\mathbb{E} e^{-T(0)} P_{n, T}(0)-D \mathbb{E} M^{n-1}+O\left(s^{\beta+\epsilon}\right),
$$

where $D$ is a constant. In fact, putting $s=0$ in the above equality and subtracting, we obtain (4.20).

It remains to prove (4.21). By (4.1), we can decompose the left hand side of (4.21) into three parts:

$$
\phi^{(n)}(s)-\mathbb{E} M^{n-1} \phi^{(n)}(s M)=\mathbb{E} e^{-T(s)} P_{n, T}(s)+\mathbb{E} e^{-T(s)}\left(-T^{\prime}(s)\right)^{n}-\left[\mathbb{E} e^{-T(s)} T^{(n)}(s)+\mathbb{E} M^{n-1} \phi^{(n)}(s M)\right] .
$$

In the following, we will show that the three terms on the right hand side of (4.22) are equal to

$$
\mathbb{E} e^{-T(0)} P_{n, T}(0)+O\left(s^{\beta+\epsilon}\right), \quad \mathbb{E} e^{-s Y_{1}}\left(-Y_{1}\right)^{n}+O\left(s^{\beta+\epsilon}\right) \quad \text { and } \quad D \mathbb{E} M^{n-1}+O\left(s^{\beta+\epsilon}\right),
$$

respectively. This together with (4.22) implies (4.21), which ends the proof of (4.20). The proof for the above three terms will be done in three steps respectively.

Step 1. We first show that

$$
\mathbb{E}\left\{e^{-T(s)} P_{n, T}(s)-e^{-T(0)} P_{n, T}(0)\right\}=O\left(s^{\beta+\epsilon}\right), \quad s \geq 0 .
$$

Recall that $P_{n, T}(s)$ is of the form

$$
P_{n, T}(s)=\sum_{a_{1}, \ldots, a_{n-1}} c\left(a_{1}, \ldots, a_{n-1}\right)\left(T^{\prime}(s)\right)^{a_{1}} \cdots\left(T^{(n-1)}(s)\right)^{a_{n-1}},
$$

where $a_{k} \in \mathbb{N}$ satisfy (3.4). So we first estimate

$$
e^{-T(s)} \prod_{k=1}^{n-1}\left(T^{(k)}(s)\right)^{a_{k}}-e^{-T(0)} \prod_{k=1}^{n-1}\left(T^{(k)}(0)\right)^{a_{k}}=I_{1}+I_{2},
$$

where

$$
\begin{aligned}
& I_{1}=e^{-T(s)}\left[\prod_{k=1}^{n-1}\left(T^{(k)}(s)\right)^{a_{k}}-\prod_{k=1}^{n-1}\left(T^{(k)}(0)\right)^{a_{k}}\right], \\
& I_{2}=\left[e^{-T(s)}-e^{-T(0)}\right] \prod_{k=1}^{n-1}\left(T^{(k)}(0)\right)^{a_{k}} .
\end{aligned}
$$


Let us show that $\left|\mathbb{E} I_{1}\right| \leq C s^{\beta+\epsilon}$. As

$$
\prod_{k=1}^{n-1} a_{k}-\prod_{k=1}^{n-1} b_{k}=\sum_{l=1}^{n-1}\left(a_{l}-b_{l}\right)\left(\prod_{k=1}^{l-1} b_{k}\right)\left(\prod_{k=l+1}^{n-1} a_{k}\right)
$$

(with the convention that the empty product is taken to be 1), we have

$$
\prod_{k=1}^{n-1}\left(T^{(k)}(s)\right)^{a_{k}}-\prod_{k=1}^{n-1}\left(T^{(k)}(0)\right)^{a_{k}}=\sum_{l=1}^{n-1}\left[\left(T^{(l)}(s)\right)^{a_{l}}-\left(T^{(l)}(0)\right)^{a_{l}}\right] \prod_{k=1}^{l-1}\left(T^{(k)}(0)\right)^{a_{k}} \cdot \prod_{k=l+1}^{n-1}\left(T^{(k)}(s)\right)^{a_{k}}
$$

Notice that for any integer $l \geq 1, a^{l}-b^{l}=(a-b)\left(\sum_{x+y=l-1} a^{x} b^{y}\right)$ (with an evident interpretation when $l=1$ ), where $x$ and $y$ are strictly positive integers. So we get

$$
\left(T^{(l)}(s)\right)^{a_{l}}-\left(T^{(l)}(0)\right)^{a_{l}}=\left[T^{(l)}(s)-T^{(l)}(0)\right] \sum_{x+y=a_{l}-1}\left(T^{(l)}(s)\right)^{x}\left(T^{(l)}(0)\right)^{y} .
$$

Since $T^{(k)}(s)=\sum_{i=1}^{N} A_{i}^{k} t^{(k)}\left(s A_{i}\right)$ and $\sup _{s \geq 0}\left|t^{(k)}(s)\right|<\infty$ for $k=1, \ldots, n-1$ (by Lemma 3.1(i)), we have

$$
\left|T^{(k)}(s)\right| \leq \sum_{i=1}^{N} A_{i}^{k}\left|t^{(k)}\left(s A_{i}\right)\right| \leq C \sum_{i=1}^{N} A_{i}^{k} \leq C Y_{1}^{k}, \quad 1 \leq k \leq n-1 .
$$

As $\left|t^{(l)}(s)-t^{(l)}(0)\right| \leq C s^{\beta+\epsilon}$ for $1 \leq l \leq n-1$ and $s \geq 0$ (it follows from (3.8) for $s \in[0,1]$, and from (3.7) for $s \geq 1$ ), we see that

$$
\begin{aligned}
\left|T^{(l)}(s)-T^{(l)}(0)\right| & \leq \sum_{i=1}^{N} A_{i}^{l}\left|t^{(l)}\left(s A_{i}\right)-t^{(l)}(0)\right| \\
& \leq C s^{\beta+\epsilon} \sum_{i=1}^{N} A_{i}^{l+\beta+\epsilon}, \quad s \geq 0, \quad 1 \leq l \leq n-1 .
\end{aligned}
$$

From (4.25)-(4.28), we obtain

$$
\begin{aligned}
\prod_{k=1}^{n-1}\left(T^{(k)}(s)\right)^{a_{k}}-\prod_{k=1}^{n-1}\left(T^{(k)}(0)\right)^{a_{k}} & =\sum_{l=1}^{n-1} O\left(s^{\beta+\epsilon}\right) \sum_{i=1}^{N} A_{i}^{l+\beta+\epsilon} \cdot\left(Y_{1}\right)^{\left(a_{l}-1\right) l} \cdot Y_{1}^{n-a_{l} l} \\
& =O\left(s^{\beta+\epsilon}\right) A^{\beta+\epsilon} \cdot Y_{1}^{n},
\end{aligned}
$$

and

$$
\left|I_{1}\right| \leq C e^{-T(s)} s^{\beta+\epsilon} A^{\beta+\epsilon} Y_{1}^{n} \leq C s^{\beta+\epsilon} A^{\beta+\epsilon} Y_{1}^{n}, \quad s \geq 0 .
$$

Notice that by Hölder's inequality,

$$
\mathbb{E} A^{\beta+\epsilon} Y_{1}^{n} \leq\left(\mathbb{E} A^{(\beta+\epsilon) p}\right)^{\frac{1}{p}} \cdot\left(\mathbb{E} Y_{1}^{n p^{*}}\right)^{\frac{1}{p^{*}}}<\infty,
$$

where $p=\frac{n+\beta-\epsilon}{\beta-\epsilon}, p^{*}=\frac{n+\beta-\epsilon}{n}$ and $\epsilon>0$ is small such that $(\beta+\epsilon) p=(\beta+\epsilon)(n+\beta-\epsilon) /(\beta-\epsilon)<n+\beta+\delta_{0}$ (recall that $\mathbb{E} Y_{1}^{n+\beta-\epsilon}<\infty$ and $\mathbb{E} A^{n+\beta+\delta_{0}}<\infty$ ). So it follows from (4.29) and (4.30) that

$$
\left|\mathbb{E} I_{1}\right| \leq C s^{\beta+\epsilon}, \quad s \geq 0 .
$$

We now consider $I_{2}$. As $a_{1} \neq n$, there exists $l \geq 2$ with $a_{l} \geq 1$, so that

$$
\left|T^{(l)}(0)\right| \leq \sum_{i=1}^{N} A_{i}^{l}\left|t^{(l)}(0)\right| \leq C \sum_{i=1}^{N} A_{i}^{l-1} A \leq C A Y_{1}^{l-1} .
$$


Noting that $\left|T^{(k)}(s)\right|=C Y_{1}^{k}$ for $1 \leq k \leq n-1$ (cf. (4.27)), we have

$$
\begin{aligned}
\prod_{k=1}^{n-1}\left|T^{(k)}(0)\right|^{a_{k}} & =\left|T^{(l)}(0)\right|^{a_{l}} \cdot \prod_{\substack{k=1 \\
k \neq l}}^{n-1}\left|T^{(k)}(0)\right|^{a_{k}} \\
& \leq C A^{a_{l}} Y_{1}^{(l-1) a_{l}} Y_{1}^{n-l a_{l}} \\
& =C A^{a_{l}} Y_{1}^{n-a_{l}} .
\end{aligned}
$$

On the other hand, for $\beta+\epsilon \in(0,1]$, as $1-e^{-x} \leq x^{\beta+\epsilon}(\forall x>0)$ and $T(s) \leq s Y_{1}$, we have

$$
1-e^{-T(s)} \leq\left(s Y_{1}\right)^{\beta+\epsilon}, \quad s \geq 0 .
$$

So

$$
\left|I_{2}\right| \leq C\left(s Y_{1}\right)^{\beta+\epsilon} A^{a_{l}} Y_{1}^{n-a_{l}} \leq C s^{\beta+\epsilon} A^{\beta+\epsilon} Y_{1}^{n} .
$$

Since $\mathbb{E} A^{\beta+\epsilon} Y_{1}^{n}<\infty$ (by (4.30)), we see that

$$
\left|\mathbb{E} I_{2}\right| \leq C s^{\beta+\epsilon}, \quad s \geq 0
$$

From (4.24), (4.31) and (4.33), we get

$$
\mathbb{E}\left\{e^{-T(s)} \prod_{k=1}^{n-1}\left(T^{(k)}(s)\right)^{a_{k}}-e^{-T(0)} \prod_{k=1}^{n-1}\left(T^{(k)}(0)\right)^{a_{k}}\right\}=O\left(s^{\beta+\epsilon}\right), \quad s \geq 0 .
$$

This gives (4.23).

Step 2. We next show that

$$
\mathbb{E} e^{-T(s)}\left(-T^{\prime}(s)\right)^{n}=\mathbb{E} e^{-s Y_{1}}\left(-Y_{1}\right)^{n}+O\left(s^{\beta+\epsilon}\right), \quad s \geq 0 .
$$

To this end, we use the decomposition

$\mathbb{E} e^{-T(s)}\left(-T^{\prime}(s)\right)^{n}-E e^{-s Y_{1}}\left(-Y_{1}\right)^{n}=\mathbb{E}\left\{e^{-T(s)}\left[\left(-T^{\prime}(s)\right)^{n}-\left(-Y_{1}\right)^{n}\right]\right\}+\mathbb{E}\left\{\left(e^{-T(s)}-e^{-s Y_{1}}\right)\left(-Y_{1}\right)^{n}\right\}(.4$

We shall prove that each of the right terms is of order $O\left(s^{\beta+\epsilon}\right)$.

As $\mathbb{E} Z^{2}<\infty$ and $\beta+\epsilon \leq 1$, we have $\left|t^{\prime}(s)-t^{\prime}(0)\right| \leq C s^{\beta+\epsilon}$ (by (3.9) of Lemma 3.1), so that

$$
\begin{aligned}
\left|T^{\prime}(s)-Y_{1}\right| & \leq \sum_{i=1}^{N} A_{i}\left|t^{\prime}\left(s A_{i}\right)-t^{\prime}(0)\right| \\
& \leq C s^{\beta+\epsilon} \sum_{i=1}^{N} A_{i}^{1+\beta+\epsilon} \leq C s^{\beta+\epsilon} A^{\beta+\epsilon} Y_{1} .
\end{aligned}
$$

Since $\left|T^{\prime}(s)\right|=\left|\sum_{i=1}^{N} A_{i} t^{\prime}\left(s A_{i}\right)\right| \leq Y_{1} \sup _{s \geq 0}\left|t^{\prime}(s)\right|$, this implies that

$$
\begin{aligned}
e^{-T(s)}\left|\left(-T^{\prime}(s)\right)^{n}-\left(-Y_{1}\right)^{n}\right| & \leq\left|T^{\prime}(s)-Y_{1}\right| \sum_{k=1}^{n-1}\left|T^{\prime}(s)\right|^{k} Y_{1}^{n-1-k} \\
& \leq C\left(\sup _{s \geq 0}\left|t^{\prime}(s)\right|+1\right)^{n} \cdot s^{\beta+\epsilon} A^{\beta+\epsilon} Y_{1}^{n} .
\end{aligned}
$$

Recalling that $\mathbb{E} A^{\beta+\epsilon} Y_{1}^{n}<\infty$, we thus obtain that

$$
\mathbb{E} e^{-T(s)}\left|\left(-T^{\prime}(s)\right)^{n}-\left(-Y_{1}\right)^{n}\right| \leq C s^{\beta+\epsilon}, \quad s \geq 0 .
$$

By Lemma 4.1,

$$
\mathbb{E}\left(e^{-T(s)}-e^{-s Y_{1}}\right) Y_{1}^{n} \leq C s^{\beta+\epsilon}, \quad s \geq 0 .
$$

Combining (4.39) and (4.40), we get (4.35). 
Step 3. We then show that for some constant $D$,

$$
\mathbb{E} e^{-T(s)} T^{(n)}(s)=-\mathbb{E} M^{n-1} \phi^{(n)}(s M)+D \mathbb{E} M^{n-1}+O\left(s^{\beta+\epsilon}\right), \quad s \geq 0 .
$$

In fact, as $0 \leq 1-e^{-T(s)} \leq(T(s))^{\beta+\epsilon} \leq s^{\beta+\epsilon} Y_{1}^{\beta+\epsilon}(\forall s>0$ and $\beta+\epsilon \leq 1)$ and $\sup _{s \geq 0}\left|t^{(n)}(s)\right|<\infty$, we see that

$$
\begin{aligned}
e^{-T(s)} T^{(n)}(s) & =\left\{\left(1+O\left(s^{\beta+\epsilon}\right) Y_{1}^{\beta+\epsilon}\right) \sum_{i=1}^{N} A_{i}^{n} t^{(n)}\left(s A_{i}\right)\right\} \\
& =\left\{\sum_{i=1}^{N} A_{i}^{n} t^{(n)}\left(s A_{i}\right)\right\}+O\left(s^{\beta+\epsilon}\right) A^{\beta+\epsilon} Y_{1}^{n} .
\end{aligned}
$$

Since $\mathbb{E} Z^{n}<\infty$ and $t^{(k)}(s)=t^{(k)}(0)+O(s)$ for $1 \leq k \leq n-1$ (by (3.8) of Lemma 3.1), from the representation form $(3.2)$ we get

$$
\phi^{(n)}(s)=-t^{(n)}(s)+D+O(s), \quad s>0,
$$

for some constant $D$ (independent of $s$ ). Since $\phi^{(n)}(s)$ and $t^{(n)}(s)$ are bounded, it follows that

$$
\phi^{(n)}(s)=-t^{(n)}(s)+D+O\left(s^{\beta+\epsilon}\right)
$$

for $\beta+\epsilon \in(0,1]$ and $s \geq 0$. Therefore,

$$
e^{-T(s)} T^{(n)}(s)=\sum_{i=1}^{N} A_{i}^{n}\left(-\phi^{(n)}\left(s A_{i}\right)+D\right)+O\left(s^{\beta+\epsilon}\right) A^{\beta+\epsilon} Y_{1}^{n}, \quad s \geq 0 .
$$

As $\mathbb{E} A^{\beta+\epsilon} Y_{1}^{n}<\infty$ (cf. (4.30)), by the dominated convergence theorem and (4.43), together with the definition of $M$ (cf. (2.10)), we obtain that

$$
\begin{aligned}
\mathbb{E} e^{-T(s)} T^{(n)}(s) & =\mathbb{E} \sum_{i=1}^{N} A_{i}^{n}\left(-\phi^{(n)}\left(s A_{i}\right)+D\right)+O\left(s^{\beta+\epsilon}\right) \mathbb{E} A^{\beta+\epsilon} Y_{1}^{n} \\
& =-\mathbb{E} M^{n-1} \phi^{(n)}(s M)+D \mathbb{E} M^{n-1}+O\left(s^{\beta+\epsilon}\right) \mathbb{E} A^{\beta+\epsilon} Y_{1}^{n} \\
& =-\mathbb{E} M^{n-1} \phi^{(n)}(s M)+D \mathbb{E} M^{n-1}+O\left(s^{\beta+\epsilon}\right), \quad s \geq 0 .
\end{aligned}
$$

This ends the proof of (4.41).

From (4.23), (4.35) and (4.41), together with (4.22), we obtain (4.21). This ends the proof of (4.20), which is equivalent to (2.12) according the the analysis at the beginning of Part (b).

The proof of Theorem 2.1 for $\alpha \in[1, \infty) \backslash\{2,3, \ldots\}$ is thus finished.

\section{Proof of Theorem 2.1: case where $\alpha \in\{2,3, \ldots\}$}

The proof of Theorem 2.1 in the case where $\alpha \in\{2,3, \ldots\}$ is similar to that in the case where $\alpha$ is not an integer, but is more delicate.

Proof of Theorem 2.1: case where $\alpha=n+1 \in\{2,3, \ldots\}$. We also distinguish two cases according to $n=1$ or $n>1$.

(a) Case $n=1$. As in the case where $\alpha \in(1,2)$, it is sufficient to prove that

$$
\mathbb{E} T^{\prime}(s)+\mathbb{E} \phi^{\prime}(s M)-\rho(2) s=O\left(s^{1+\epsilon}\right), \quad s \geq 0 .
$$

Using (2.10) with $f(x)=-\phi^{\prime}(s x)$, we have

$$
\begin{aligned}
\mathbb{E} T^{\prime}(s)+\mathbb{E} \phi^{\prime}(s M)-\rho(2) s & =\mathbb{E}\left\{\sum_{i=1}^{N} A_{i}\left(e^{t\left(s A_{i}\right)}-1\right)\left(-\phi^{\prime}\left(s A_{i}\right)\right)-\sum_{i=1}^{N} s A_{i}^{2}\right\} \\
& =s \mathbb{E} \sum_{i=1}^{N} A_{i}^{2}\left\{\frac{1-\phi\left(s A_{i}\right)}{s A_{i}}\left[-\frac{\phi^{\prime}\left(s A_{i}\right)}{\phi\left(s A_{i}\right)}\right]-1\right\} \\
& =K_{3}+K_{4},
\end{aligned}
$$


where

$$
\begin{aligned}
& K_{3}=s \mathbb{E} \sum_{i=1}^{N} A_{i}^{2}\left\{\frac{1-\phi\left(s A_{i}\right)-s A_{i}}{s A_{i}}\left[-\frac{\phi^{\prime}\left(s A_{i}\right)}{\phi\left(s A_{i}\right)}\right]\right\}, \\
& K_{4}=s \mathbb{E} \sum_{i=1}^{N} A_{i}^{2}\left\{-\frac{\phi^{\prime}\left(s A_{i}\right)}{\phi\left(s A_{i}\right)}-1\right\}=s \mathbb{E} \sum_{i=1}^{N} A_{i}^{2}\left(t^{\prime}\left(s A_{i}\right)-1\right) .
\end{aligned}
$$

As $\left|1-e^{-x}-x\right| \leq C x^{1+\epsilon}(x \geq 0)$ and $\mathbb{E} Z^{1+\epsilon}<\infty$, we see that $|1-\phi(s)-s| \leq C \mathbb{E} Z^{1+\epsilon} s^{1+\epsilon}$, and

$$
\left|1-\phi\left(s A_{i}\right)-s A_{i}\right| \leq C \mathbb{E} Z^{1+\epsilon} s^{1+\epsilon} A_{i}^{1+\epsilon} .
$$

Combining this with the fact that $\sup _{s \geq 0}\left|-\frac{\phi^{\prime}(s)}{\phi(s)}\right|<\infty$ (by Lemma 3.1(i)), we have

$$
\left|K_{3}\right| \leq C s^{1+\epsilon} \mathbb{E} \sum_{i=1}^{N} A_{i}^{2+\epsilon}=C s^{1+\epsilon} \rho(2+\epsilon), \quad s \geq 0 .
$$

We now consider $K_{4}$. Recalling that $\left|t^{\prime}(s)-t^{\prime}(0)\right| \leq C s^{\epsilon}$, we have

$$
\left|t^{\prime}\left(s A_{i}\right)-1\right| \leq C s^{\epsilon} A_{i}^{\epsilon},
$$

and

$$
\left|K_{4}\right| \leq C s^{1+\epsilon} \mathbb{E} \sum_{i=1}^{N} A_{i}^{2+\epsilon}=C s^{1+\epsilon} \rho(2+\epsilon), \quad s \geq 0 .
$$

It follows from (5.2) and (5.3) that (5.1) holds.

(b) Case $n>1$. As in the case where $\alpha>2$ is not an integer, it is sufficient to prove that

$$
\phi^{(n)}(s)-\mathbb{E} M^{n-1} \phi^{(n)}(s M)=(-1)^{n} \mathbb{E} e^{-s Y_{1}} Y_{1}^{n}+c_{0}+c_{n} s+O\left(s^{1+\epsilon}\right), \quad s \geq 0,
$$

where $c_{0}$ and $c_{n}$ are non-negative constants. In fact, putting $s=0$ in the above equality and subtracting, and noticing that $\psi_{n}(s)=(-1)^{n+1}\left[\phi^{(n)}(s)-\phi^{(n)}(0)\right]$ and $g_{n}(s)=-\mathbb{E}\left[Y_{1}^{n}\left(e^{-s Y_{1}}-1\right)\right]$, we obtain $(2.12)$.

In the following, we will show that the three terms on the right hand side of (4.22) are respectively

$$
\mathbb{E} e^{-T(0)} P_{n, T}(0)+D_{1} s+O\left(s^{1+\epsilon}\right), \quad \mathbb{E} e^{-s Y_{1}}\left(-Y_{1}\right)^{n}+(-1)^{n} D_{2} s+O\left(s^{1+\epsilon}\right) \quad \text { and } \quad D_{3}+D_{4} s+O\left(s^{1+\epsilon}\right),
$$

where $D_{i}(i=1,2,3,4)$ are non-negative constants. This together with (4.22) imply (5.4). The proof for the above three terms will be done in three steps respectively.

Step 1. We first show that for some constant $D_{1}$,

$$
\mathbb{E}\left\{e^{-T(s)} P_{n, T}(s)-e^{-T(0)} P_{n, T}(0)\right\}=D_{1} s+O\left(s^{1+\epsilon}\right), \quad s \geq 0 .
$$

We begin with the estimation of

$$
e^{-T(s)} \prod_{k=1}^{n-1}\left(T^{(k)}(s)\right)^{a_{k}}-e^{-T(0)} \prod_{k=1}^{n-1}\left(T^{(k)}(0)\right)^{a_{k}}=J_{1}+J_{2},
$$

where

$$
\begin{aligned}
& J_{1}=e^{-T(s)}\left[\prod_{k=1}^{n-1}\left(T^{(k)}(s)\right)^{a_{k}}-\prod_{k=1}^{n-1}\left(T^{(k)}(0)\right)^{a_{k}}\right], \\
& J_{2}=\left[e^{-T(s)}-e^{-T(0)}\right] \prod_{k=1}^{n-1}\left(T^{(k)}(0)\right)^{a_{k}} .
\end{aligned}
$$


We first consider $J_{1}$. As $t^{(l)}(s)=t^{(l)}(0)+t^{(l+1)}(0) s+O\left(s^{1+\epsilon}\right)$ for $1 \leq l \leq n-1$ (by (3.8) of Lemma 3.1), we see that

$$
\begin{aligned}
T^{(l)}(s)-T^{(l)}(0) & =\sum_{i=1}^{N} A_{i}^{l}\left[t^{(l)}\left(s A_{i}\right)-t^{(l)}(0)\right] \\
& =\sum_{i=1}^{N} A_{i}^{l}\left[t^{(l+1)}(0) s A_{i}+O\left(s^{1+\epsilon}\right) A_{i}^{1+\epsilon}\right] \\
& =t^{(l+1)}(0) s \sum_{i=1}^{N} A_{i}^{l+1}+O\left(s^{1+\epsilon}\right) \sum_{i=1}^{N} A_{i}^{l+1+\epsilon} .
\end{aligned}
$$

Since $t^{(k)}(s)=t^{(k)}(0)+O\left(s^{\epsilon}\right)$ for $k=1,2, \ldots, n-1$, we have

$$
T^{(k)}(s)=\sum_{i=1}^{N} A_{i}^{k} t^{(k)}\left(s A_{i}\right)=t^{(k)}(0) \sum_{i=1}^{N} A_{i}^{k}+O\left(s^{\epsilon}\right) \sum_{i=1}^{N} A_{i}^{k+\epsilon} .
$$

Therefore, by (4.25), we obtain

$$
\prod_{k=1}^{n-1}\left(T^{(k)}(s)\right)^{a_{k}}-\prod_{k=1}^{n-1}\left(T^{(k)}(0)\right)^{a_{k}}=J_{3} s+O\left(s^{1+\epsilon}\right) A^{1+\epsilon} Y_{1}^{n},
$$

where

$$
J_{3}=\sum_{l=1}^{n-1}\left[t^{(l+1)}(0) \sum_{i=1}^{N} A_{i}^{l+1}\right]\left[t^{(l)}(0) \sum_{i=1}^{N} A_{i}^{l}\right]^{a_{l}-1} \prod_{\substack{k=1 \\ k \neq l}}^{n-1}\left[t^{(k)}(0) \sum_{i=1}^{N} A_{i}^{k}\right]^{a_{k}} .
$$

By Hölder's inequality,

$$
\mathbb{E} A^{1+\epsilon} Y_{1}^{n+\epsilon} \leq\left(\mathbb{E} A^{(1+\epsilon) p}\right)^{1 / p}\left(\mathbb{E} Y_{1}^{n p^{*}}\right)^{1 / p^{*}}<\infty,
$$

where $p=\frac{\alpha-\epsilon}{1-2 \epsilon}, p^{*}=\frac{\alpha-\epsilon}{n+\epsilon}$ and $\epsilon>0$ small enough such that $(1+\epsilon) p=(1+\epsilon)(\alpha-\epsilon) /(1-2 \epsilon) \leq \alpha+\delta_{0}$. As $J_{3}$ is dominated by $O(1) A Y_{1}^{n}$ with $\mathbb{E}\left[A Y_{1}^{n}\right]<\infty$ (by (5.7)) and $e^{-T(s)}=1+O\left(s^{\epsilon}\right) Y_{1}^{\epsilon}, J_{1}=J_{3} s+$ $O\left(s^{1+\epsilon}\right)\left(A Y_{1}^{n+\epsilon}+A^{1+\epsilon} Y_{1}^{n+\epsilon}\right)$, we see that

$$
\mathbb{E} J_{1}=s \mathbb{E} J_{3}+O\left(s^{1+\epsilon}\right), \quad s \geq 0 .
$$

Since $1-e^{-x}-x=O\left(x^{1+\epsilon}\right)(0<\epsilon \leq 1, x \geq 0)$ and $T(s)=s Y_{1}+O\left(s^{1+\epsilon}\right) Y_{1}^{1+\epsilon}$, we have

$$
e^{-T(s)}-1=-s Y_{1}+O\left(s^{1+\epsilon}\right) Y_{1}^{1+\epsilon} \text {. }
$$

Using this together with $T^{(k)}(0)=t^{(k)}(0) \sum_{i=1}^{N} A_{i}^{k}$, we get

$$
\begin{aligned}
J_{2} & =\left[-s Y_{1}+O\left(s^{1+\epsilon}\right) Y_{1}^{1+\epsilon}\right] \prod_{k=1}^{n-1}\left(t^{(k)}(0) \sum_{i=1}^{N} A_{i}^{k}\right)^{a_{k}} \\
& =-s J_{4}+O\left(s^{1+\epsilon}\right) Y_{1}^{n} A^{1+\epsilon},
\end{aligned}
$$

where

$$
J_{4}=Y_{1} \prod_{k=0}^{n-1}\left(t^{(k)}(0) \sum_{i=1}^{N} A_{i}^{k}\right)^{a_{k}}
$$

is dominated by $O(1) A Y_{1}^{n}$ with $\mathbb{E} A Y_{1}^{n}<\infty$ (by (5.7)). So by (5.7),

$$
\mathbb{E} J_{2}=-s \mathbb{E} J_{4}+O\left(s^{1+\epsilon}\right), \quad s \geq 0 .
$$

It follows from (5.8) and (5.9) that (5.5) holds with $D_{1}=\mathbb{E} J_{3}-\mathbb{E} J_{4}$.

Step 2. We next show that, for some constant $D_{2}$ and $s \geq 0$,

$$
\mathbb{E} e^{-T(s)}\left(T^{\prime}(s)\right)^{n}-\mathbb{E} e^{-s Y_{1}} Y_{1}^{n}-D_{2} s=O\left(s^{1+\epsilon}\right) .
$$


Notice that (5.10) holds evidently for $s \in(1, \infty)$ (as $\left|T^{\prime}(s)\right| \leq C Y_{1}$ and $\mathbb{E} Y_{1}^{n}<\infty$ ), so we only need to prove it for $s \in[0,1]$. To prove this, it is sufficient to show the following two estimations:

$$
\begin{aligned}
\mathbb{E} e^{-T(s)}\left[\left(T^{\prime}(s)\right)^{n}-Y_{1}^{n}\right] & =D_{2} s+O\left(s^{1+\epsilon}\right), \quad s \in[0,1], \\
\mathbb{E}\left[e^{-T(s)}-e^{-s Y_{1}}\right] Y_{1}^{n} & =O\left(s^{1+\epsilon}\right), \quad s \in[0,1] .
\end{aligned}
$$

We first show (5.11). Notice that

$$
\left(T^{\prime}(s)\right)^{n}-Y_{1}^{n}=\left(T^{\prime}(s)-Y_{1}\right)\left[\sum_{k=0}^{n-1}\left(T^{\prime}(s)\right)^{k} Y_{1}^{n-1-k}\right] .
$$

Since $T^{\prime}(s)=Y_{1}+O\left(s^{\epsilon}\right) A^{\epsilon} Y_{1}$ (cf. (4.37)), writing $\bar{A}=\max (A, 1)$, we have for $s \in[0,1]$,

$$
\begin{aligned}
\sum_{k=0}^{n-1}\left(T^{\prime}(s)\right)^{k} Y_{1}^{n-1-k} & =\sum_{k=0}^{n-1}\left[1+O\left(s^{\epsilon}\right) A^{\epsilon}\right]^{k} Y_{1}^{n-1} \\
& =\sum_{k=0}^{n-1}\left[1+O\left(s^{\epsilon}\right) \bar{A}^{k \epsilon}\right] Y_{1}^{n-1} \\
& =\left[n+O\left(s^{\epsilon}\right) \bar{A}^{(n-1) \epsilon}\right] Y_{1}^{n-1},
\end{aligned}
$$

where the second equality holds since for $0 \leq k \leq n-1$,

$$
\left[1+O\left(s^{\epsilon}\right) \bar{A}^{\epsilon}\right]^{k}-1=\sum_{j=1}^{k}\left(\begin{array}{c}
k \\
j
\end{array}\right) O\left(s^{j \epsilon}\right) \bar{A}^{j \epsilon}=O\left(s^{\epsilon}\right) \bar{A}^{k \epsilon},
$$

using the fact that $s \in[0,1]$ and $\bar{A} \geq 1$ imply $s^{j \epsilon} \leq s^{\epsilon}$ and $\bar{A}^{j \epsilon} \leq \bar{A}^{k \epsilon}$ for each $1 \leq j \leq k$. As $t^{\prime}(s)=1+t^{\prime \prime}(0) s+O\left(s^{1+\epsilon}\right)(s \geq 0)$, we see that

$$
\begin{aligned}
T^{\prime}(s)-Y_{1} & =\sum_{i=1}^{N} A_{i}\left(t^{\prime}\left(s A_{i}\right)-1\right) \\
& =t^{\prime \prime}(0) s \sum_{i=1}^{N} A_{i}^{2}+O\left(s^{1+\epsilon}\right) \sum_{i=1}^{N} A_{i}^{2+\epsilon} .
\end{aligned}
$$

From (5.13), (5.14) and (5.15), we obtain that for $s \in[0,1]$,

$$
\begin{aligned}
\left(T^{\prime}(s)\right)^{n}-Y_{1}^{n}= & {\left[t^{\prime \prime}(0) s \sum_{i=1}^{N} A_{i}^{2}+O\left(s^{1+\epsilon}\right) \sum_{i=1}^{N} A_{i}^{2+\epsilon}\right]\left[n+O\left(s^{\epsilon}\right) \bar{A}^{(n-1) \epsilon}\right] Y_{1}^{n-1} } \\
= & n t^{\prime \prime}(0) s \sum_{i=1}^{N} A_{i}^{2} Y_{1}^{n-1}+O\left(s^{1+\epsilon}\right) \sum_{i=1}^{N} A_{i}^{2+\epsilon} Y_{1}^{n-1} \\
& +O\left(s^{1+\epsilon}\right) \sum_{i=1}^{N} A_{i}^{2} \bar{A}^{(n-1) \epsilon} Y_{1}^{n-1}+O\left(s^{1+2 \epsilon}\right) \sum_{i=1}^{N} A_{i}^{2+\epsilon} \bar{A}^{(n-1) \epsilon} Y_{1}^{n-1} .
\end{aligned}
$$

Recalling that $Y_{1}=\sum_{i=1}^{N} A_{i}$ and $\bar{A} \geq 1$, we have

$$
\begin{aligned}
\sum_{i=1}^{N} A_{i}^{2+\epsilon} Y_{1}^{n-1} & \leq A^{1+\epsilon} Y_{1}^{n} \leq \bar{A}^{1+n \epsilon} Y_{1}^{n}, \\
\sum_{i=1}^{N} A_{i}^{2} \bar{A}^{(n-1) \epsilon} Y_{1}^{n-1} & \leq \bar{A}^{1+n \epsilon} Y_{1}^{n}, \\
\sum_{i=1}^{N} A_{i}^{2+\epsilon} \bar{A}^{(n-1) \epsilon} Y_{1}^{n-1} & \leq \bar{A}^{1+n \epsilon} Y_{1}^{n} .
\end{aligned}
$$


Hence from (5.16), we get

$$
\left(T^{\prime}(s)\right)^{n}-Y_{1}^{n}=n t^{\prime \prime}(0) s \sum_{i=1}^{N} A_{i}^{2} Y_{1}^{n-1}+O\left(s^{1+\epsilon}\right) \bar{A}^{1+n \epsilon} Y_{1}^{n} .
$$

Since $e^{-x}=1+O\left(x^{\epsilon}\right)(0<\epsilon \leq 1, x \geq 0)$ and $0 \leq T(s) \leq s Y_{1}$ (cf. (2.8)), together with (5.17), we see that for $s \in[0,1]$,

$$
\begin{aligned}
& e^{-T(s)}\left[\left(T^{\prime}(s)\right)^{n}-Y_{1}^{n}\right] \\
= & {\left[1+O\left(s^{\epsilon}\right) Y_{1}^{\epsilon}\right]\left[n t^{\prime \prime}(0) s \sum_{i=1}^{N} A_{i}^{2} Y_{1}^{n-1}+O\left(s^{1+\epsilon}\right) \bar{A}^{1+n \epsilon} Y_{1}^{n}\right] } \\
= & n t^{\prime \prime}(0) s \sum_{i=1}^{N} A_{i}^{2} Y_{1}^{n-1}+O\left(s^{1+\epsilon}\right)\left[\bar{A} Y_{1}^{n+\epsilon}+\bar{A}^{1+n \epsilon} Y_{1}^{n}+\bar{A}^{1+n \epsilon} Y_{1}^{n+\epsilon}\right] \\
= & n t^{\prime \prime}(0) s \sum_{i=1}^{N} A_{i}^{2} Y_{1}^{n-1}+O\left(s^{1+\epsilon}\right) \bar{A}^{1+n \epsilon}\left[Y_{1}^{n}+Y_{1}^{n+\epsilon}\right]
\end{aligned}
$$

Notice that

$$
\mathbb{E} \sum_{i=1}^{n} A_{i}^{2} Y_{1}^{n-1} \leq \mathbb{E} A Y_{1}^{n} \leq\left(\mathbb{E} A^{p}\right)^{1 / p}\left(\mathbb{E} Y_{1}^{n p^{*}}\right)^{1 / p^{*}}<\infty,
$$

where $p=\alpha+\delta_{0}, p^{*}=\frac{\alpha+\delta_{0}}{\alpha+\delta_{0}-1}$, so that $n p^{*}<\alpha$ and that

$$
\begin{aligned}
\mathbb{E} \bar{A}^{1+n \epsilon} Y_{1}^{n} & \leq C\left[\mathbb{E} A^{1+n \epsilon} Y_{1}^{n}+\mathbb{E} Y_{1}^{n}\right] \\
& \leq C\left\{\left[\mathbb{E} A^{[1+n \epsilon] q}\right]^{1 / q}\left[\mathbb{E} Y_{1}^{n q^{*}}\right]^{1 / q^{*}}+\mathbb{E} Y_{1}^{n}\right\}<\infty, \\
\mathbb{E} \bar{A}^{1+n \epsilon} Y_{1}^{n+\epsilon} & \leq C\left[\mathbb{E} A^{1+n \epsilon} Y_{1}^{n+\epsilon}+\mathbb{E} Y_{1}^{n+\epsilon}\right] \\
& \leq C\left\{\left[\mathbb{E} A^{[1+n \epsilon] q}\right]^{1 / q}\left[\mathbb{E} Y_{1}^{(n+\epsilon) q^{*}}\right]^{1 / q^{*}}+\mathbb{E} Y_{1}^{n+\epsilon}\right\}<\infty,
\end{aligned}
$$

where $q=\frac{\alpha+\delta_{0}}{1+n \epsilon}, q^{*}=\frac{\alpha+\delta_{0}}{\left(\alpha+\delta_{0}\right)-[1+n \epsilon]}, \delta_{0}$ and $\epsilon$ are small enough. By the dominated convergence theorem, it follows from (5.18) that (5.11) holds with $D_{2}=n t^{\prime \prime}(0) \mathbb{E} \sum_{i=1}^{N} A_{i}^{2} Y_{1}^{n-1}$.

We now prove (5.12). By the same argument as in the proof of (4.7) (choosing $\beta=1$ ), we see that if $C s^{1-\epsilon} A^{1-\epsilon} \leq 1 / 2$, then $T(s) \geq s Y_{1} / 2$, so that

$$
e^{-T(s)}-e^{-s Y_{1}} \leq C e^{-s Y_{1} / 2} s^{2-\epsilon} A^{1-\epsilon} Y_{1} .
$$

Therefore,

$$
\begin{aligned}
\left(e^{-T(s)}-e^{-s Y_{1}}\right) Y_{1}^{n} \mathbf{1}_{\left\{C s^{1-\epsilon} A^{1-\epsilon} \leq 1 / 2\right\}} & \leq C e^{-s Y_{1} / 2}\left(s Y_{1}\right)^{1-2 \epsilon} s^{1+\epsilon} A^{1-\epsilon} Y_{1}^{n+2 \epsilon} \\
& \leq C s^{1+\epsilon} A^{1-\epsilon} Y_{1}^{n+2 \epsilon} .
\end{aligned}
$$

On the other hand,

$$
\begin{aligned}
\left(e^{-T(s)}-e^{-s Y_{1}}\right) Y_{1}^{n} \mathbf{1}_{\left\{C s^{1-\epsilon} A^{1-\epsilon}>1 / 2\right\}} & \leq C Y_{1}^{n}\left(s^{1-\epsilon} A^{1-\epsilon}\right)^{(1+\epsilon) /(1-\epsilon)} \\
& \leq C s^{1+\epsilon} A^{1-\epsilon} Y_{1}^{n+2 \epsilon} .
\end{aligned}
$$

Hence, we have shown that

$$
\left(e^{-T(s)}-e^{-s Y_{1}}\right) Y_{1}^{n} \leq C s^{1+\epsilon} A^{1-\epsilon} Y_{1}^{n+2 \epsilon} .
$$

As $\mathbb{E} A^{1-\epsilon} Y_{1}^{n+\epsilon}<\infty$ (cf. (5.7)), this gives (5.12) which ends the proof of (5.10).

Step 3. We then show that, for some constants $D_{3}$ and $D_{4}$,

$$
\mathbb{E} e^{-T(s)} T^{(n)}(s)+\mathbb{E} M^{n-1} \phi^{(n)}(s M)=D_{3}+D_{4} s+O\left(s^{1+\epsilon}\right), \quad s \geq 0 .
$$


Notice that $0<x-1+e^{-x} \leq C x^{1+\epsilon}(\forall x>0)$ and $T(s) \leq s Y_{1}$, we have

$$
e^{-T(s)}=1-T(s)+O\left(s^{1+\epsilon}\right) Y_{1}^{1+\epsilon} .
$$

As $\mathbb{E} Z^{n}<\infty$, we have $\sup _{s \geq 0}\left|t^{(k)}(s)\right|<\infty$ for $0 \leq k \leq n$, and $t^{(k)}(s)=t^{(k)}(0)+t^{(k+1)}(0) s+O\left(s^{1+\epsilon}\right)$ for $0 \leq k \leq n-1$ (by Lemma 3.1(i)). So from the representation form (3.2) and $\sup _{s>0}\left|\phi^{(n)}\right| \leq \mathbb{E} Z^{n}<\infty$, we see that for $s \geq 0$,

$$
t(s)=s+O\left(s^{1+\epsilon}\right) \quad \text { and } \quad t^{(n)}(s)=-\phi^{(n)}(s)+D+D^{\prime} s+O\left(s^{1+\epsilon}\right) .
$$

Hence from the definition of $T^{(n)}(s)$, we obtain that

$$
\begin{aligned}
e^{-T(s)} T^{(n)}(s)= & \sum_{i=1}^{N} A_{i}^{n} t^{(n)}\left(s A_{i}\right)-\sum_{i=1}^{N} t\left(s A_{i}\right) \cdot \sum_{i=1}^{N} A_{i}^{n} t^{(n)}\left(s A_{i}\right)+O\left(s^{1+\epsilon}\right) Y_{1}^{1+\epsilon} \sum_{i=1}^{N} A_{i}^{n} t^{(n)}\left(s A_{i}\right) \\
= & -\sum_{i=1}^{N} A_{i}^{n} \phi^{(n)}\left(s A_{i}\right)+D \sum_{i=1}^{N} A_{i}^{n}+s\left[D^{\prime} \sum_{i=1}^{N} A_{i}^{n+1}-Y_{1} \sum_{i=1}^{N} A_{i}^{n} t^{(n)}\left(s A_{i}\right)\right] \\
& +O\left(s^{1+\epsilon}\right)\left[\sum_{i=1}^{N} A_{i}^{n+1+\epsilon}+\left(\sum_{i=1}^{N} A_{i}^{1+\epsilon}+Y_{1}^{1+\epsilon}\right) \sum_{i=1}^{N} A_{i}^{n} t^{(n)}\left(s A_{i}\right)\right] .
\end{aligned}
$$

Since $\mathbb{E} A^{1+\epsilon} Y_{1}^{n}<\infty($ by $(5.7))$ and $\sup _{s>0}\left|t^{(n)}(s)\right|<\infty$ (by (3.7)), we see that

$$
\mathbb{E}\left[\sum_{i=1}^{N} A_{i}^{n+1}+Y_{1} \sum_{i=1}^{N} A_{i}^{n} \mid t^{(n)}\left(s A_{i}\right)\right] \leq\left(1+\sup _{s>0}\left|t^{(n)}(s)\right|\right) \mathbb{E} A^{1+\epsilon} Y_{1}^{n-\epsilon}<\infty
$$

and

$$
\mathbb{E}\left[\sum_{i=1}^{N} A_{i}^{n+1+\epsilon}+\left(\sum_{i=1}^{N} A_{i}^{1+\epsilon}+Y_{1}^{1+\epsilon}\right) \sum_{i=1}^{N} A_{i}^{n}\left|t^{(n)}\left(s A_{i}\right)\right|\right] \leq\left[1+2 \sup _{s>0}\left|t^{(n)}(s)\right|\right] \mathbb{E} A^{1+\epsilon} Y_{1}^{n}<\infty .
$$

Therefore,

$$
\begin{aligned}
\mathbb{E} e^{-T(s)} T^{(n)}(s) & =-\mathbb{E} \sum_{i=1}^{N} A_{i}^{n} \phi^{(n)}\left(s A_{i}\right)+D_{3}+D_{4} s+O\left(s^{1+\epsilon}\right) \\
& =-\mathbb{E} M^{n-1} \phi^{(n)}(s M)+D_{3}+D_{4} s+O\left(s^{1+\epsilon}\right),
\end{aligned}
$$

where $D_{3}=D \rho(n)$ and $D_{4}=\mathbb{E}\left[D^{\prime} \sum_{i=1}^{N} A_{i}^{n+1}-Y_{1} \sum_{i=1}^{N} A_{i}^{n} t^{(n)}\left(s A_{i}\right)\right]$. This ends the proof of (5.19).

From (5.5), (5.10) and (5.19), together with (4.22), we obtain (5.4). This ends the proof of (2.12) according to the the analysis at the beginning of Part (b).

The proof of Theorem 2.1 for $\alpha \in\{2,3, \ldots\}$ is thus finished.

\section{Proof of Theorem 1.1}

Recall that without loss of generality, we can take $\ell$ as the form (1.10). Therefore, by Potter's theorem (cf. [11], p.25), for every $\epsilon>0$, there is $C=C(\epsilon)>0$ such that

$$
\frac{\ell(y)}{\ell(x)} \leq C \max \left\{\left(\frac{y}{x}\right)^{\epsilon},\left(\frac{x}{y}\right)^{\epsilon}\right\}, \quad x, y>0 .
$$

Proof of Theorem 1.1. We distinguish two cases according to $\alpha \in(1, \infty) \backslash \mathbb{N}$ or $\alpha \in\{2,3, \ldots\}$.

(i) Case $\alpha \in(1, \infty) \backslash \mathbb{N}$. By Proposition 2.1, it suffices to show that the following two assertions are equivalent:

$$
\begin{array}{ll}
h_{n}(s) \sim s^{\alpha} \ell(1 / s) & (s \rightarrow 0), \\
\phi_{n}(s) \sim \frac{s^{\alpha} \ell(1 / s)}{1-\rho(\alpha)} & (s \rightarrow 0) .
\end{array}
$$


Step 1. We first show that (6.3) implies (6.2). By Theorem 2.2, we have

$$
\phi_{n}(s)-\mathbb{E} M^{-1} \phi_{n}(s M)=h_{n}(s)+O\left(s^{\alpha+\epsilon}\right), \quad s \geq 0 .
$$

Notice that $\frac{\phi_{n}(s)}{s^{n}}$ is bounded and $\ell(1 / s)=1$ for $s>1$. Therefore (6.3) implies

$$
\left|\phi_{n}(s)\right| \leq C s^{\alpha} \ell(1 / s), \quad s>0
$$

and

$$
\begin{aligned}
M^{-1} \phi_{n}(s M) & \sim M^{-1}(s M)^{\alpha} \ell\left(\frac{1}{s M}\right) \cdot \frac{1}{1-\rho(\alpha)} \\
& \sim M^{\alpha-1} s^{\alpha} \ell\left(\frac{1}{s}\right) \cdot \frac{1}{1-\rho(\alpha)}
\end{aligned}
$$

By (6.5) and Potter's theorem, we see that

$$
\begin{aligned}
\left|\frac{M^{-1} \phi_{n}(s M)}{s^{\alpha} \ell(1 / s)}\right| & \leq C\left(\frac{M^{-1}(s M)^{\alpha} \ell\left(\frac{1}{s M}\right)}{s^{\alpha} \ell(1 / s)}\right) \\
& =C\left(M^{\alpha-1} \ell\left(\frac{1}{s M}\right) / \ell(1 / s)\right) \\
& \leq C \max \left(M^{\alpha-1+\epsilon}, M^{\alpha-1-\epsilon}\right)
\end{aligned}
$$

(recall that $C>0$ may differ from line to line). Therefore by the dominated convergence theorem, we have

$$
\lim _{s \rightarrow 0} \frac{\mathbb{E} M^{-1} \phi_{n}(s M)}{s^{\alpha} \ell(1 / s)}=\frac{\mathbb{E} M^{\alpha-1}}{1-\rho(\alpha)}=\frac{\rho(\alpha)}{1-\rho(\alpha)} .
$$

As $\frac{O\left(s^{\epsilon}\right)}{\ell(1 / s)}=o(1)(s \rightarrow 0)$, it follows from (6.4) that $\lim _{s \rightarrow 0} \frac{h_{n}(s)}{s^{\alpha} \ell(1 / s)}=1$. So (6.2) holds.

Step 2. We next show that (6.2) implies (6.3). Recall that by Theorem 2.2,

$$
\phi_{n}(s)-\mathbb{E} M^{-1} \phi_{n}(s M)=h_{n}(s)+O\left(s^{\alpha+\epsilon}\right), \quad s \geq 0 .
$$

Therefore $\hat{\phi}_{n}(s):=\phi_{n}(s) / s^{\alpha}$ and $\hat{h}_{n}(s):=h_{n}(s) / s^{\alpha}$ satisfy

$$
\hat{\phi}_{n}(s)-\mathbb{E} M^{\alpha-1} \hat{\phi}_{n}(s M)=\hat{h}_{n}(s)+O\left(s^{\epsilon}\right), \quad s \geq 0 .
$$

Let

$$
\hat{\phi}(s)=\hat{\phi}_{n}(s)-\mathbb{E} M^{\alpha-1} \hat{\phi}_{n}(s M)
$$

Then

$$
\hat{\phi}(s)=\hat{\phi}_{n}(s)-\rho(\alpha) \mathbb{E} \hat{\phi}_{n}(s B),
$$

where $B \geq 0$ is a random variable whose distribution is determined by

$$
\mathbb{E} f(B)=\frac{1}{\rho(\alpha)} \mathbb{E} M^{\alpha-1} f(M)
$$

for all measurable functions $f \geq 0$. Let $B_{i}$ be independent copies of $B$. Then by $(6.9), \forall i \geq 1$,

$$
\rho^{i-1}(\alpha) \hat{\phi}\left(s B_{1} \cdots B_{i-1}\right)=\rho^{i-1}(\alpha) \hat{\phi}_{n}\left(s B_{1} \cdots B_{i-1}\right)-\rho^{i}(\alpha) \mathbb{E}_{i} \hat{\phi}_{n}\left(s B_{1} \cdots B_{i-1} B_{i}\right)
$$

where $\mathbb{E}_{i}$ denotes the expectation with respect to $B_{i}$, and $B_{1} \cdots B_{i-1}=1$ if $i=1$. Taking expectation and then summing for $i$ from 0 to $k-1$, we obtain

$$
\sum_{i=0}^{k-1} \rho^{i}(\alpha) \mathbb{E} \hat{\phi}\left(s B_{1} \cdots B_{i}\right)=\hat{\phi}_{n}(s)-\rho^{k}(\alpha) \mathbb{E} \hat{\phi}_{n}\left(s B_{1} \cdots B_{k}\right) .
$$

Let us now prove that

$$
\lim _{k \rightarrow \infty} \rho^{k}(\alpha) \mathbb{E} \hat{\phi}_{n}\left(s B_{1} \cdots B_{k}\right)=0
$$


As $\mathbb{E} Z^{\alpha-\epsilon}<\infty$, by $(3.10)$,

$$
\left|\hat{\phi}_{n}(s)\right| \leq C s^{-\epsilon},
$$

so

$$
\begin{aligned}
\mathbb{E} \rho^{k}(\alpha)\left|\hat{\phi}_{n}\left(s B_{1} \cdots B_{k}\right)\right| & \leq C \mathbb{E} \rho^{k}(\alpha)\left(s^{-\epsilon} B_{1}^{-\epsilon} \cdots B_{k}^{-\epsilon}\right) \\
& =C s^{-\epsilon} \rho^{k}(\alpha)\left(\mathbb{E} B^{-\epsilon}\right)^{k} \\
& =C s^{-\epsilon} \rho^{k}(\alpha)\left(\frac{\mathbb{E} M^{\alpha-1-\epsilon}}{\rho(\alpha)}\right)^{k} \\
& =C s^{-\epsilon} \rho^{k}(\alpha-\epsilon) .
\end{aligned}
$$

As $\rho(\alpha-\epsilon)<1$, this prove (6.13). So by (6.12), we obtain

$$
\sum_{i=0}^{\infty} \rho^{i}(\alpha) \mathbb{E} \hat{\phi}\left(s B_{1} \cdots B_{i}\right)=\hat{\phi}_{n}(s) .
$$

Notice that by the condition (6.2) and the equation (6.8), we have as $s \rightarrow 0$,

$$
\hat{\phi}(s) \sim \ell(1 / s)
$$

and

$$
\hat{\phi}\left(s B_{1} \cdots B_{i}\right) \sim \ell\left(\frac{1}{s B_{1} \cdots B_{i}}\right) \sim \ell(1 / s) .
$$

By Potter's bound (cf. (6.1)) and the dominated convergence theorem, this yields

$$
\lim _{s \rightarrow 0} \frac{\mathbb{E} \hat{\phi}\left(s B_{1} \cdots B_{i}\right)}{\ell(1 / s)}=1 .
$$

From (6.16),(6.17) and the dominated convergence theorem, we obtain

$$
\lim _{s \rightarrow 0} \frac{\hat{\phi}_{n}(s)}{\ell(1 / s)}=\sum_{i=0}^{\infty} \rho^{i}(\alpha)=\frac{1}{1-\rho(\alpha)},
$$

which is just the desired result (6.3). Here the use of the dominated convergence theorem is justified by the fact that there is a sequence of non-negative numbers $\left(a_{i}\right)$ with $\sum_{i=1}^{\infty} a_{i}<\infty$ such that for all $i \geq 1$ and all $s \in[0,1]$,

$$
\frac{\rho^{i}(\alpha) \mathbb{E} \hat{\phi}\left(s B_{1} \cdots B_{i}\right)}{\ell(1 / s)} \leq a_{i} .
$$

In fact, when $s B_{1} \cdots B_{i} \leq 1$, as $\left|\frac{\hat{\phi}(s)}{\ell(1 / s)}\right| \leq C$ for all $s \in[0,1]$, we have by Potter's bound (cf. (6.1)) that

$$
\begin{aligned}
\frac{\rho^{i}(\alpha) \mathbb{E}\left|\hat{\phi}\left(s B_{1} \cdots B_{i}\right)\right|}{\ell(1 / s)} & =\rho^{i}(\alpha) \mathbb{E}\left[\frac{\left|\hat{\phi}\left(s B_{1} \cdots B_{i}\right)\right|}{\ell\left(\left(s B_{1} \cdots B_{i}\right)^{-1}\right)} \cdot \frac{\ell\left(\left(s B_{1} \cdots B_{i}\right)^{-1}\right)}{\ell\left(s^{-1}\right)}\right] \\
& \leq C \rho^{i}(\alpha) \mathbb{E}\left[\left(B_{1} \cdots B_{i}\right)^{\epsilon}+\left(B_{1} \cdots B_{i}\right)^{-\epsilon}\right] \\
& =C \rho^{i}(\alpha)\left[\left(\mathbb{E} B^{\epsilon}\right)^{i}+\left(\mathbb{E} B^{-\epsilon}\right)^{i}\right],
\end{aligned}
$$

where $C>0$ differs from line to line. When $s B_{1} \cdots B_{i}>1$, as $\frac{\ell(1)}{\ell\left(\left(s B_{1} \cdots B_{i}\right)^{-1}\right)} \leq C\left(s B_{1} \cdots B_{i}\right)^{\epsilon}$ (by $\left.(6.1)\right)$, and $\left|\hat{\phi}\left(s B_{1} \cdot B_{i}\right)\right| \leq C[1+\rho(\alpha-\epsilon)]\left(s B_{1} \cdots B_{i}\right)^{-\epsilon}$ (which is derived from (6.14) and the definition of $\hat{\phi}(\cdot)$ ), we have that

$$
\begin{aligned}
\frac{\rho^{i}(\alpha) \mathbb{E}\left|\hat{\phi}\left(s B_{1} \cdots B_{i}\right)\right|}{\ell(1 / s)} & =\rho^{i}(\alpha) \mathbb{E}\left[\frac{\left|\hat{\phi}\left(s B_{1} \cdots B_{i}\right)\right|}{\ell(1)} \cdot \frac{\ell(1)}{\ell\left(\left(s B_{1} \cdots B_{i}\right)^{-1}\right)} \cdot \frac{\ell\left(\left(s B_{1} \cdots B_{i}\right)^{-1}\right)}{\ell\left(s^{-1}\right)}\right] \\
& \leq C \rho^{i}(\alpha) \mathbb{E}\left\{\left(s B_{1} \cdots B_{i}\right)^{-\epsilon} \cdot\left(s B_{1} \cdots B_{i}\right)^{\epsilon} \cdot\left[\left(B_{1} \cdots B_{i}\right)^{\epsilon}+\left(B_{1} \cdots B_{i}\right)^{-\epsilon}\right]\right\} \\
& =C \rho^{i}(\alpha)\left[\mathbb{E}\left(B_{1} \cdots B_{i}\right)^{-\epsilon}+\mathbb{E}\left(B_{1} \cdots B_{i}\right)^{\epsilon}\right] \\
& =C \rho^{i}(\alpha)\left[\left(\mathbb{E} B^{-\epsilon}\right)^{i}+\left(\mathbb{E} B^{\epsilon}\right)^{i}\right],
\end{aligned}
$$


where $C>0$ differs from line to line. It should be pointed out that the $O(1)$ s above are not depending on $i$. From the distribution of $B$ (cf. (6.11)), we have

$$
\rho^{i}(\alpha)\left[\left(\mathbb{E} B^{-\epsilon}\right)^{i}+\left(\mathbb{E} B^{\epsilon}\right)^{i}\right]=\left(\mathbb{E} M^{\alpha-1-\epsilon}\right)^{i}+\left(\mathbb{E} M^{\alpha-1+\epsilon}\right)^{i}=\rho^{i}(\alpha-\epsilon)+\rho^{i}(\alpha+\epsilon),
$$

which is summable due to the facts that $\rho(\alpha-\epsilon)<1$ and $\rho(\alpha+\epsilon)<1$. Hence we have shown (6.19). This ends the proof of Step 2. Thus the proof for the case $\alpha \in(1, \infty) \backslash \mathbb{N}$ is finished.

(ii) Case $\alpha \in\{2,3, \ldots\}$. In this case we write

$$
\hat{h}_{n-1}(s)=\frac{h_{n-1}(s)}{s^{n}}, \quad \hat{\phi}_{n-1}(s)=\frac{\phi_{n-1}(s)}{s^{n}}, \quad n=2,3, \ldots
$$

(this time we have $\alpha=(n-1)+1$, which corresponds to the preceding case with $n$ replaced by $n-1$ and $\beta=1$ ). By Proposition 2.2, we only need to prove the equivalence between the following two statements: for any fixed $\lambda>1$,

$$
\begin{aligned}
\hat{H}_{n-1}(s):=\hat{h}_{n-1}(s)-\hat{h}_{n-1}(\lambda s) & \sim \frac{\ln \lambda}{(n-1) !} \ell(1 / s) \quad(s \rightarrow 0), \\
\hat{\Phi}_{n-1}(s):=\hat{\phi}_{n-1}(s)-\hat{\phi}_{n-1}(\lambda s) & \sim \frac{\ln \lambda}{(n-1) !}(1-\rho(n))^{-1} \ell(1 / s) \quad(s \rightarrow 0) .
\end{aligned}
$$

Recall that by Theorem 2.2,

$$
\phi_{n-1}(s)-\mathbb{E} M^{-1} \phi_{n-1}(s M)=h_{n-1}(s)+\frac{c_{n-1}}{n !} s^{n}+O\left(s^{n+\epsilon}\right), \quad s \geq 0,
$$

so that

$$
\hat{\phi}_{n-1}(s)-\mathbb{E} M^{n-1} \hat{\phi}_{n-1}(s M)=\hat{h}_{n-1}(s)+\frac{c_{n-1}}{n !}+O\left(s^{\epsilon}\right), \quad s \geq 0 .
$$

Evaluating the equality at $\lambda s$ and subtracting, we obtain

$$
\hat{\Phi}_{n-1}(s)-\mathbb{E} M^{n-1} \hat{\Phi}_{n-1}(s M)=\hat{H}_{n-1}(s)+O\left(s^{\epsilon}\right), \quad s \geq 0 .
$$

Using (6.25) instead of (6.8), the same argument as in Case (i) shows that (6.21) and (6.22) are equivalent. This ends the proof of Theorem 1.1.

\section{Proof of Theorem 1.2}

Before giving the proof of Theorem 1.2, we first show two lemmas. The first lemma states that the main term $\mathbb{E}\left[e^{-T(s)}-1+T(s)\right]$ of the right-hand-side of (2.15) behaves like $h_{1}$.

Lemma 7.1 If $\rho\left(1+\delta_{0}\right)<\infty$ for some $\delta_{0}>0$, then we have for $\epsilon \in(0,1)$ and $s \geq 0$,

$$
h_{1}(s) \geq \mathbb{E}\left[e^{-T(s)}-1+T(s)\right]=h_{1}((1-\epsilon) s)+O\left(s^{1+\delta_{0}}\right) .
$$

Proof. Let $H(x)=e^{-x}-1+x(x \geq 0)$. Then we have

$$
\begin{aligned}
\mathbb{E} H\left(s Y_{1}\right) & =\mathbb{E}\left[e^{-s Y_{1}}-1+s Y_{1}\right]=h_{1}(s), \\
\mathbb{E} H(T(s)) & =\mathbb{E}\left[e^{-T(s)}-1+T(s)\right] .
\end{aligned}
$$

For $\epsilon \in(0,1)$, there exists $s_{0}=s_{0}(\epsilon)>0$ such that $t(s) \geq(1-\epsilon) s$ for all $s \in\left[0, s_{0}\right]$. Hence for $s \in\left[0, s_{0}\right]$, we have

$$
T(s) \geq \sum_{i=1}^{N}(1-\epsilon) A_{i} s \mathbf{1}_{\left\{A_{i} s \leq s_{0}\right\}}=(1-\epsilon) s \sum_{i=1}^{N} A_{i} \mathbf{1}_{\left\{A_{i} s \leq s_{0}\right\}} .
$$

As $H(x)$ is increasing on $(0, \infty)$, from $(2.8)$ and $(7.2)$ we see that

$$
\begin{aligned}
& \mathbb{E} H(T(s)) \leq \mathbb{E} H\left(s Y_{1}\right)=h_{1}(s), \\
& \mathbb{E} H(T(s)) \geq \mathbb{E} H\left((1-\epsilon) s \sum_{i=1}^{n} A_{i} \mathbf{1}_{\left\{A_{i} s \leq s_{0}\right\}}\right) .
\end{aligned}
$$


Notice that by the mean value theorem, $0 \leq H(b)-H(a) \leq b-a$ if $0 \leq a \leq b$. Hence

$$
\begin{aligned}
0 & \leq H\left((1-\epsilon) s Y_{1}\right)-H\left((1-\epsilon) s \sum_{i=1}^{N} A_{i} \mathbf{1}_{\left\{A_{i} s \leq s_{0}\right\}}\right) \\
& \leq(1-\epsilon) s \sum_{i=1}^{N} A_{i} \mathbf{1}_{\left\{A_{i} s>s_{0}\right\}} \\
& \leq(1-\epsilon) s^{1+\delta_{0}} \sum_{i=1}^{N} A_{i}^{1+\delta_{0}} / s_{0}^{\delta_{0}} .
\end{aligned}
$$

Taking expectation, we see that

$$
\begin{aligned}
0 & \leq \mathbb{E}\left[H\left((1-\epsilon) s Y_{1}\right)-H\left((1-\epsilon) s \sum_{i=1}^{N} A_{i} \mathbf{1}_{\left\{A_{i} s \leq s_{0}\right\}}\right)\right] \\
& \leq(1-\epsilon) s_{0}^{-\delta_{0}} \rho\left(1+\delta_{0}\right) s^{1+\delta_{0}} .
\end{aligned}
$$

As $\mathbb{E} H\left((1-\epsilon) s Y_{1}\right)=h_{1}((1-\epsilon) s)$, it follows from (7.3), (7.4) and (7.5) that (7.1) holds.

The second lemma is a relation between $\tilde{\ell}$ and $\ell$. It shows that we can present $\tilde{\ell}$ as a sum of $\ell$ composed with a random walk.

Lemma 7.2 Let $\left(X_{i}\right)_{i \geq 0}$ be independent and identically distributed with $\mu=\mathbb{E} X_{1} \in(0, \infty)$ and $\mathbb{E} e^{-\delta_{0} X_{1}}<$ $\infty$ for some $\delta_{0}>0$. Write $S_{n}=X_{1}+\cdots+X_{n}$. Let $\ell \in R_{0}$ be non-increasing with $\int_{1}^{\infty} \ell(t) d t / t<\infty$. Then as $x \rightarrow \infty$

$$
\frac{1}{\tilde{\ell}(x)} \sum_{n=0}^{\infty} \ell\left(x e^{S_{n}}\right) \rightarrow \frac{1}{\mu} \quad \text { a.s. and in } L^{1},
$$

where $\tilde{\ell}$ is defined in (1.17).

Proof. Without loss of generality, we assume that $\ell$ is given by (1.10). We first estimate the inferior limit of $\frac{\sum_{n=0}^{\infty} \mathbb{E} \ell\left(x e^{S_{n}}\right)}{\tilde{\ell}(x)}$. As $\mathbb{E} X_{1}=\mu \in(0, \infty)$, by the law of large numbers we have $S_{n} / n \rightarrow \mu$ a.s. For $\epsilon>0$, there exists almost surely a finite $N_{\epsilon}$ such that $S_{n}$ lies between $n(\mu \pm \epsilon)$ for $n \geq N_{\epsilon}$. Since $\ell$ is non-increasing and $\int_{1}^{\infty} \frac{\ell(x)}{x} d x<\infty$, we have $\lim _{x \rightarrow \infty} \ell(x)=0$. Using this together with $\ell^{\prime}(x)=\ell(x) \epsilon(x) / x$ (cf. (1.10)), we obtain that

$$
\ell(x)=-\int_{x}^{\infty} \ell^{\prime}(t) d t=\int_{x}^{\infty} \ell(t)(-\epsilon(t)) d t / t .
$$

Therefore

$$
\frac{\ell(x)}{\tilde{\ell}(x)}=\frac{\int_{x}^{\infty} \ell(t)(-\epsilon(t)) d t / t}{\int_{x}^{\infty} \ell(t) d t / t} \leq \sup _{t \in[x, \infty)}(-\epsilon(t)) \rightarrow 0 \quad(x \rightarrow \infty)
$$

so that

$$
\ell(x)=o(\tilde{\ell}(x)) \quad(x \rightarrow \infty) .
$$

As $\ell\left(x e^{S_{n}}\right) \sim \ell(x)$, it follows that a.s.

$$
\sum_{n=0}^{N_{\epsilon}-1} \ell\left(x e^{S_{n}}\right)=o(\tilde{\ell}(x)) \quad(x \rightarrow \infty) .
$$

On the other hand, for $n \geq N_{\epsilon}$, as $\ell$ is non-increasing, we have a.s.

$$
\sum_{n=N_{\epsilon}}^{\infty} \ell\left(x e^{n(\mu+\epsilon)}\right) \leq \sum_{n=N_{\epsilon}}^{\infty} \ell\left(x e^{S_{n}}\right) \leq \sum_{n=N_{\epsilon}}^{\infty} \ell\left(x e^{n(\mu-\epsilon)}\right) .
$$

Notice that for any $c>0$,

$$
\sum_{n=N_{\epsilon}+1}^{\infty} \ell\left(x e^{n c}\right) \leq \int_{N_{\epsilon}}^{\infty} \ell\left(x e^{c t}\right) d t \leq \sum_{n=N_{\epsilon}}^{\infty} \ell\left(x e^{n c}\right),
$$


and that the integral above (the middle term) is

$$
\frac{1}{c} \int_{x e^{c N_{\epsilon}}}^{\infty} \ell(u) d u / u=\frac{1}{c} \tilde{\ell}\left(x e^{c N_{\epsilon}}\right) \sim \frac{1}{c} \tilde{\ell}(x) \quad(x \rightarrow \infty) .
$$

Therefore

$$
\sum_{n=N_{\epsilon}}^{\infty} \ell\left(x e^{n c}\right) \sim \frac{1}{c} \tilde{\ell}(x) \quad(x \rightarrow \infty) .
$$

It follows from (7.8), (7.9) and (7.10) that a.s.

$$
\frac{\sum_{n=0}^{\infty} \ell\left(x e^{S_{n}}\right)}{\tilde{\ell}(x)} \rightarrow \frac{1}{\mu} \quad(x \rightarrow \infty) .
$$

So by Fatou's lemma,

$$
\liminf _{x \rightarrow \infty} \frac{\sum_{n=0}^{\infty} \mathbb{E} \ell\left(x e^{S_{n}}\right)}{\tilde{\ell}(x)} \geq \mathbb{E} \liminf _{x \rightarrow \infty} \frac{\sum_{n=0}^{\infty} \ell\left(x e^{S_{n}}\right)}{\tilde{\ell}(x)}=\frac{1}{\mu} .
$$

We next consider the superior limit of $\frac{\sum_{n=0}^{\infty} \mathbb{E} \ell\left(x e^{S_{n}}\right)}{\tilde{\ell}(x)}$. We divide $S_{n}$ into two parts according to $\left\{S_{n} \leq\right.$ $n(\mu-\epsilon)\}$ or $\left\{S_{n}>n(\mu-\epsilon)\right\}$, so that

$$
\sum_{n=0}^{\infty} \mathbb{E} \ell\left(x e^{S_{n}}\right)=\sum_{n=0}^{\infty} \mathbb{E} \ell\left(x e^{S_{n}}\right) \mathbf{1}_{\left\{S_{n} \leq n(\mu-\epsilon)\right\}}+\sum_{n=0}^{\infty} \mathbb{E} \ell\left(x e^{S_{n}}\right) \mathbf{1}_{\left\{S_{n}>n(\mu-\epsilon)\right\}} .
$$

As $\ell$ is non-increasing, the second term is bounded by

$$
\sum_{n=0}^{\infty} \ell\left(x e^{n(\mu-\epsilon)}\right) \sim \frac{1}{\mu-\epsilon} \tilde{\ell}(x) \quad(x \rightarrow \infty)
$$

(cf. (7.10) with $c=\mu-\epsilon$ ). We now deal with the first term in the right hand side of (7.13). Write $\bar{X}_{i}=X_{i}-\mu$ and $\bar{S}_{n}=\bar{X}_{1}+\cdots+\bar{X}_{n}=S_{n}-n \mu$. By Potter's theorem, for any $\delta>0$, we have

$$
\begin{aligned}
\mathbb{E} \ell\left(x e^{S_{n}}\right) \mathbf{1}_{\left\{S_{n} \leq n(\mu-\epsilon)\right\}} & \leq A_{\delta} \ell(x) \mathbb{E}\left(e^{\delta S_{n}}+e^{-\delta S_{n}}\right) \mathbf{1}_{\left\{S_{n} \leq n(\mu-\epsilon)\right\}} \\
& \leq A_{\delta} \ell(x) \mathbb{E}\left(e^{n \mu \delta}+e^{-\delta S_{n}}\right) \mathbf{1}_{\left\{\bar{S}_{n} \leq-n \epsilon\right\}},
\end{aligned}
$$

where $A_{\delta}>0$ is a constant depending on $\delta$.

We shall prove that thanks to the condition $\mathbb{E} e^{-\delta_{0} X_{1}}<\infty$, we have for $\epsilon>0$,

$$
\mathbb{P}\left(\bar{S}_{n} \leq-n \epsilon\right) \leq e^{-c n}
$$

for some $c=c(\epsilon)>0$ and all $n \geq 1$. In fact, as $\left(X_{i}\right)$ are independent and identically distributed, we have for all $\delta>0$

$$
\begin{aligned}
\mathbb{P}\left(\bar{S}_{n} \leq-n \epsilon\right) & =\mathbb{P}\left(e^{-\delta \bar{S}_{n}} \geq e^{n \delta \epsilon}\right) \\
& \leq e^{-n \delta \epsilon} \mathbb{E} e^{-\delta \bar{S}_{n}} \\
& =\left[e^{-\delta \epsilon} \mathbb{E} e^{-\delta \bar{X}_{1}}\right]^{n}
\end{aligned}
$$

Let $\Lambda(\delta)=\ln \mathbb{E} e^{-\delta \bar{X}_{1}}$. By the dominated convergence theorem, it can be easily seen that $\frac{d}{d \delta} \mathbb{E} e^{-\delta \bar{X}_{1}}=$ $\mathbb{E}\left[-\bar{X}_{1} e^{-\delta \bar{X}_{1}}\right]$, so that

$$
\Lambda^{\prime}(\delta)=\frac{\mathbb{E}\left[-\bar{X}_{1} e^{-\delta \bar{X}_{1}}\right]}{\mathbb{E} e^{-\delta \bar{X}_{1}}} \rightarrow-\mathbb{E}\left[\bar{X}_{1}\right]=0 \quad(\delta \rightarrow 0) .
$$

Hence

$$
\lim _{\delta \rightarrow 0} \frac{\Lambda(\delta)}{\delta}=\lim _{\delta \rightarrow 0} \Lambda^{\prime}(\delta)=0
$$


Therefore, for $\delta>0$ small enough, we have $\Lambda(\delta)<\delta \epsilon$, which is equivalent to

$$
\mathbb{E} e^{-\delta \bar{X}_{1}}<e^{\delta \epsilon}
$$

So from (7.17), we obtain (7.16) with $c=-\ln \left[\frac{\mathbb{E} e^{-\delta \bar{x}_{1}}}{e^{\delta \epsilon}}\right]>0$.

By the Cauchy-Schwartz inequality, it follows from (7.15) and (7.16) that

$$
\begin{aligned}
\mathbb{E} \ell\left(x e^{S_{n}}\right) \mathbf{1}_{\left\{S_{n} \leq n(\mu-\epsilon)\right\}} & \leq A_{\delta} \ell(x)\left\{e^{n \mu \delta} \mathbb{P}\left(\bar{S}_{n} \leq-n \epsilon\right)+\left[\mathbb{E} e^{-2 \delta S_{n}}\right]^{1 / 2}\left[\mathbb{P}\left(\bar{S}_{n} \leq-n \epsilon\right)\right]^{1 / 2}\right\} \\
& \leq A_{\delta} \ell(x)\left\{\left[e^{\mu \delta} \cdot e^{-c}\right]^{n}+\left[\mathbb{E} e^{-2 \delta X_{1}} \cdot e^{-c}\right]^{n / 2}\right\}
\end{aligned}
$$

As $\mathbb{E} e^{-2 \delta X_{1}}<\infty$ for $\delta \in\left[0, \delta_{0} / 2\right]$, we can choose $\delta>0$ small enough such that

$$
e^{\mu \delta} \cdot e^{-c}<1 \quad \text { and } \quad \mathbb{E} e^{-2 \delta X_{1}} \cdot e^{-c}<1 .
$$

Hence from (7.20) and (7.7), we see that

$$
\begin{aligned}
\sum_{n=1}^{\infty} \mathbb{E} \ell\left(x e^{S_{n}}\right) \mathbf{1}_{\left\{S_{n} \leq n(\mu-\epsilon)\right\}} & \leq A_{\delta} \ell(x) \sum_{n=1}^{\infty}\left\{\left[e^{\mu \delta} \cdot e^{-c}\right]^{n}+\left[\mathbb{E} e^{-2 \delta X_{1}} \cdot e^{-c}\right]^{n / 2}\right\} \\
& \leq C \ell(x)=o(\tilde{\ell}(x)) .
\end{aligned}
$$

It follows from (7.13), (7.14) and (7.22) that

$$
\sum_{n=0}^{\infty} \mathbb{E} \ell\left(x e^{S_{n}}\right) \leq o(\tilde{\ell}(x))+\frac{1}{\mu-\epsilon} \tilde{\ell}(x),
$$

which implies that

$$
\limsup _{x \rightarrow \infty} \frac{\sum_{n=0}^{\infty} \mathbb{E} \ell\left(x e^{S_{n}}\right)}{\tilde{\ell}(x)} \leq \frac{1}{\mu-\epsilon} .
$$

Letting $\epsilon \rightarrow 0$, we have

$$
\limsup _{x \rightarrow \infty} \frac{\sum_{n=0}^{\infty} \mathbb{E} \ell\left(x e^{S_{n}}\right)}{\tilde{\ell}(x)} \leq \frac{1}{\mu} .
$$

Combining this with (7.12), we obtain

$$
\lim _{x \rightarrow \infty} \frac{1}{\tilde{\ell}(x)} \sum_{n=0}^{\infty} \mathbb{E} \ell\left(x e^{S_{n}}\right)=\frac{1}{\mu} .
$$

By Scheffé's theorem, (7.11) and (7.24) give (7.6).

Proof of Theorem 1.2. By Proposition 2.1 (with $n=1$ and $\beta=0$ ), we just need to show that

$$
h_{1}(s) \sim s \ell(1 / s) \quad(s \rightarrow 0)
$$

implies

$$
\phi_{1}(s) \sim \mu^{-1} s \tilde{\ell}(1 / s) \quad(s \rightarrow 0),
$$

where $\tilde{\ell}$ is defined in (1.17). Notice that if $\ell_{1}(x) \sim \ell_{2}(x)(x \rightarrow \infty)$, then $\int_{x}^{\infty} \frac{\ell_{1}(t)}{t} d t \sim \int_{x}^{\infty} \frac{\ell_{2}(t)}{t} d t(x \rightarrow \infty)$ by l'Hôpital's rule. Therefore we can suppose that $\ell(1 / s)=h_{1}(s) / s, s>0$, which is non-decreasing, so that $\ell(x)$ is non-increasing.

Recall the definitions of $\hat{\phi}_{1}(s)$ and $\hat{\phi}(s)$ in the proof of Theorem 1.1(i) (with $\left.\alpha=1\right)$ :

$$
\hat{\phi}_{1}(s)=\phi_{1}(s) / s \text { and } \hat{\phi}(s)=\hat{\phi}_{1}(s)-\mathbb{E} \hat{\phi}_{1}(s M) .
$$

Corresponding to $(6.12)$, we get

$$
\sum_{i=0}^{k-1} \mathbb{E} \hat{\phi}\left(s M_{1} \cdots M_{i}\right)=\hat{\phi}_{1}(s)-\mathbb{E} \hat{\phi}_{1}\left(s M_{1} \cdots M_{k}\right),
$$


where $\left\{M_{i}\right\}$ are independent copies of $M$. Notice that $0<1-e^{-x}<x(\forall x>0)$, we see that $0 \leq \phi_{1}(s)=$ $\mathbb{E}\left(e^{-s Z}-1+s Z\right) \leq 2 s \mathbb{E} Z=2 s$, and hence

$$
\sup _{s>0}\left|\hat{\phi}_{1}(s)\right|<\infty .
$$

Therefore, by the dominated convergence theorem and the fact that $M_{1} \cdots M_{k} \rightarrow 0$ (which follows from the law of large numbers), we conclude that

$$
\lim _{k \rightarrow \infty} \mathbb{E} \hat{\phi}_{1}\left(s M_{1} \cdots M_{k}\right)=0 .
$$

So letting $k \rightarrow \infty$ in (7.27), we obtain

$$
\sum_{n=0}^{\infty} \mathbb{E} \hat{\phi}\left(s M_{1} \cdots M_{n}\right)=\hat{\phi}_{1}(s)
$$

By Lemma 7.1 and Theorem 2.2, we see that

$$
\begin{aligned}
& \hat{\phi}(s) \leq \frac{h_{1}(s)}{s}+O\left(s^{\epsilon}\right), \quad s \geq 0, \\
& \hat{\phi}(s) \geq \frac{h_{1}((1-\delta) s)}{s}+O\left(s^{\epsilon}\right), \quad s \geq 0,
\end{aligned}
$$

for all $\delta \in(0,1)$. From $(7.28)$ and $(7.29)$, we have for all $\epsilon>0$,

$$
\begin{aligned}
\hat{\phi}_{1}(s) & \leq \sum_{n=0}^{\infty}\left[\mathbb{E} \frac{h_{1}\left(s M_{1} \cdots M_{n}\right)}{s M_{1} \cdots M_{n}}+O\left(\mathbb{E}\left(s M_{1} \cdots M_{n}\right)^{\epsilon}\right)\right] \\
& =\sum_{n=0}^{\infty}\left[\mathbb{E} \ell\left(\frac{1}{s M_{1} \cdots M_{n}}\right)+O\left(s^{\epsilon}\right) \mathbb{E}\left(M_{1} \cdots M_{n}\right)^{\epsilon}\right] .
\end{aligned}
$$

Since (1.14) holds for $\alpha=1$ and some $\delta_{0}>0$, we have $\rho(1+\delta)<\infty$ for some $\delta>0$ small enough (cf. $(1.15))$. Hence $\rho(x)$ is convex on $(1,1+\delta)$ with $\rho^{\prime}(1)<0$, and we can find some $\epsilon>0$ small enough such that $\rho(1+\epsilon)<1$. By the definition of $M$ (cf. (2.10)), we obtain that $\mathbb{E}\left(M_{1} \cdots M_{n}\right)^{\epsilon}=\left(\mathbb{E} \sum_{i=1}^{N} A_{i}^{1+\epsilon}\right)^{n}=$ $\rho^{n}(1+\epsilon)$ and

$$
\sum_{n=0}^{\infty} \mathbb{E}\left(M_{1} \cdots M_{n}\right)^{\epsilon}=\sum_{n=0}^{\infty} \rho^{n}(1+\epsilon)=\frac{1}{1-\rho(1+\epsilon)} .
$$

Write $M_{n}=e^{-X_{n}}$ and $S_{n}=X_{1}+\cdots+X_{n}$. So $\left\{X_{n}\right\}$ are independent and identically distributed, with $\mathbb{E} X_{1}=-\mathbb{E} \ln M_{1}=-\rho^{\prime}(1)=\mu \in(0, \infty)$ and $\mathbb{E} e^{-\delta_{0} X_{1}}=\mathbb{E} M_{1}^{\delta_{0}}=\rho\left(1+\delta_{0}\right)<\infty$. By Lemma 7.2 , we have

$$
\begin{aligned}
\sum_{n=0}^{\infty} \mathbb{E} \ell\left(\frac{1}{s M_{1} \cdots M_{n}}\right) & =\sum_{n=0}^{\infty} \mathbb{E} \ell\left(e^{S_{n}} / s\right) \\
& \sim \mu^{-1} \tilde{\ell}(1 / s) \quad(s \rightarrow 0) .
\end{aligned}
$$

Notice that $\tilde{\ell}$ is a function slowly varying at $\infty$, it follows from (7.31), (7.32) and (7.33) that

$$
\limsup _{s \rightarrow 0} \frac{\hat{\phi}_{1}(s)}{\tilde{\ell}(1 / s)} \leq \lim _{s \rightarrow 0}\left[\frac{1}{\mu}+\frac{O\left(s^{\epsilon}\right)}{\tilde{\ell}(1 / s)}\right]=\mu^{-1} .
$$

On the other hand, from (7.28) and (7.30), we have for $\delta \in(0,1)$,

$$
\begin{aligned}
\hat{\phi}_{1}(s) & \geq \sum_{n=0}^{\infty}\left[\mathbb{E} \frac{h_{1}\left((1-\delta) s M_{1} \cdots M_{n}\right)}{s M_{1} \cdots M_{n}}+O\left(s^{\epsilon}\right) \mathbb{E}\left(M_{1} \cdots M_{n}\right)^{\epsilon}\right] \\
& =\sum_{n=0}^{\infty}\left[(1-\delta) \mathbb{E} \ell\left(\frac{1}{(1-\delta) s M_{1} \cdots M_{n}}\right)+O\left(s^{\epsilon}\right) \mathbb{E}\left(M_{1} \cdots M_{n}\right)^{\epsilon}\right] \\
& =\sum_{n=0}^{\infty}\left[(1-\delta) \mathbb{E} \ell\left(e^{S_{n}} /[(1-\delta) s]\right)+O\left(s^{\epsilon}\right) \mathbb{E}\left(M_{1} \cdots M_{n}\right)^{\epsilon}\right] .
\end{aligned}
$$


Using again Lemma 7.2, we have

$$
\sum_{n=0}^{\infty} \mathbb{E} \ell\left(e^{S_{n}} /[(1-\delta) s]\right) \sim \mu^{-1} \tilde{\ell}(1 /[(1-\delta) s]) \sim \mu^{-1} \tilde{\ell}(1 / s) \quad(s \rightarrow 0),
$$

where the last step holds since the slow variation of $\ell$ implies that of $\tilde{\ell}$. It follows from (7.32), (7.35) and (7.36) that

$$
\liminf _{s \rightarrow 0} \frac{\hat{\phi}_{1}(s)}{\tilde{\ell}(1 / s)} \geq \frac{1-\delta}{\mu} .
$$

Letting $\delta \rightarrow 0$, we see that

$$
\liminf _{s \rightarrow 0} \frac{\hat{\phi}_{1}(s)}{\tilde{\ell}(1 / s)} \geq \mu^{-1}
$$

From (7.34) and (7.38), we obtain

$$
\lim _{s \rightarrow 0} \frac{\hat{\phi}_{1}(s)}{\tilde{\ell}(1 / s)}=\mu^{-1},
$$

which is equivalent to (7.26). This ends the proof of Theorem 1.2.

Remark 7.1 In Theorem 1.2, we fail to show the equivalence between (1.18) and (1.19), which can be interpreted to the equivalence between (7.25) and (7.26). In our approach, to show the implication (7.25) $\Rightarrow$ (7.26), we use (7.28) (together with Lemma 7.2) to transfer information on $h_{1}$ (corresponding to $\hat{\phi}$ ) to that on $\phi_{1}$ (corresponding to $\hat{\phi}_{1}$ ). The difficult for establishing the inverse implication $(7.26) \Rightarrow(7.25)$ is that from (7.28), we cannot transfer the information from $\hat{\phi}_{1}$ to $\hat{\phi}$ (that is, from $\phi_{1}$ to $h_{1}$ ).

\section{Acknowledgements}

The authors are very grateful to the reviewers for very valuable comments and remarks which helped us much to improve significantly the manuscript. The work has been partially supported by the National Natural Science Foundation of China (Grants nos. 11601019, 11571052 and 11731012), by the Scientific Research Project of Beijing Municipal Education (Grant no. SQKM201610011006), by Hunan Provincial Natural Science Foundation (China, Grant no. 2017JJ2271), and by the French Government Program "Investissements d'Avenir" ANR-11-LABX-0020-01 (Centre Henri Lebesgue).

\section{Appendix}

Proof of Proposition 2.2. Write $F$ for the distribution function of $X$. Define $F_{1}, \cdots, F_{n+1}$ by letting $F_{1}=F$ and

$$
1-F_{k+1}(x)= \begin{cases}\int_{x}^{\infty}\left[1-F_{k}(t)\right] d t, & x \geq 0 \\ 1-F_{k+1}(0), & x<0\end{cases}
$$

if $1 \leq k \leq n$. By induction, it can be easily shown that when $\mu_{n}=\mathbb{E} X^{n}<\infty$, we have for $k=0, \ldots, n$,

$$
1-F_{k+1}(x)=\frac{\mathbb{E}\left[(X-x)^{k} \mathbf{1}_{\{X>x\}}\right]}{k !}, \quad x \geq 0 ;
$$

in particular, $1-F_{k+1}(x)$ is bounded by $1-F_{k+1}(0)=\mu_{k} / k !$. By definition, for $1 \leq k \leq n$,

$$
F_{k+1}(x)-F_{k+1}(0)=\int_{0}^{x}\left[1-F_{k}(t)\right] d t, \quad x \geq 0 .
$$

In other words, for $k=1, \cdots, n, F_{k+1}(x)-F_{k+1}(0)$ is the distribution function of the measure concentrated on $(0, \infty)$ of density $1-F_{k}(t)$ with respect to the Lebesgue measure, with mass $1-F_{k+1}(0)=\mu_{k} / k !$. Define

$$
F_{n+2}(x)=\int_{0}^{x}\left[1-F_{n+1}(t)\right] d t, \quad x \geq 0
$$


and $F_{n+2}(x)=0$ for $x<0$, namely, $F_{n+2}(x)$ is the distribution function of the measure concentrated on $(0, \infty)$ of density $1-F_{n+1}(t)$ with respect to the Lebesgue measure. By induction, it can be easily checked that for $k=0, \cdots, n$,

$$
\hat{f}_{k}(s)=\int_{[0, \infty)} e^{-s t} d\left(F_{k+2}(x)-F_{k+2}(0)\right)=\int_{[0, \infty)} e^{-s t}\left[1-F_{k+1}(t)\right] d t
$$

We first show that (2.3) implies (2.4). As $1-F(x) \sim x^{-(n+1)} \ell(x)(x \rightarrow \infty)$, by induction we have

$$
1-F_{k+1}(x) \sim \frac{x^{-(n+1-k)}}{n \cdots(n-k+1)} \ell(x), \quad k=0, \ldots, n,
$$

with the convention that $n \cdots(n-k+1)=1$ when $k=0$. Therefore, for any $\lambda>0$, we have

$$
\begin{aligned}
F_{n+2}(\lambda x)-F_{n+2}(x) & =\int_{x}^{\lambda x}\left[1-F_{n+1}(t)\right] d t \\
& \sim \int_{x}^{\lambda x} \frac{\ell(t)}{n !} \frac{d t}{t} \sim \frac{\ell(x)}{n !} \ln \lambda, \quad x \rightarrow \infty,
\end{aligned}
$$

where the last equivalence is due to the Uniform Convergence Theorem (see [11, Theorem 1.2.1]). Hence we obtain

$$
\lim _{x \rightarrow \infty} \frac{F_{n+2}(\lambda x)-F_{n+2}(x)}{\ell(x)}=\frac{1}{n !} \ln \lambda, \quad \forall \lambda>0 .
$$

As $\hat{f}_{n}$ is the Laplace -Stieltjes transform of $F_{n+2}$, it follows from (A.2) and de Haan's theorem (see [15] or $[11$, Theorem 3.9.1]) that

$$
\lim _{s \rightarrow \infty} \frac{\hat{f}_{n}\left(\frac{1}{\lambda s}\right)-\hat{f}_{n}\left(\frac{1}{s}\right)}{\ell(s)}=\frac{1}{n !} \ln \lambda, \quad \forall \lambda>0
$$

which gives (2.4).

We now show that $(2.4)$ implies $(2.3)$. Since $\hat{f}_{n}(\cdot)$ is the Laplace-Stieltjes transform of $F_{n+2}(\cdot)$, again by de Haan's theorem, we see that (A.3) holds true, which implies that

$$
\lim _{x \rightarrow \infty} \frac{F_{n+2}(\lambda x)-F_{n+2}(x)}{F_{n+2}(e x)-F_{n+2}(x)}=\ln \lambda, \quad \forall \lambda>0
$$

So by the lemma of [15], we see that $1-F_{n+1}(x)$, which is the derivative of $F_{n+2}(x)$, is a regularly varying function of index -1 . Differentiating $n$ times the function $1-F_{n+1}(x)$, we know that $1-F_{1}(x)$ varies regularly with index $-(n+1)$, that is,

$$
1-F_{1}(x) \sim x^{-(n+1)} \ell_{1}(x) \quad(x \rightarrow \infty),
$$

where $\ell_{1}(\cdot)$ is a function slowly varying at $\infty$. Using this and the implication " $(2.3) \Rightarrow(2.4)$ ", we see that (2.4) holds also with $\ell$ replaced by $\ell_{1}$. Thus $\ell_{1}(x) \sim \ell(x)(x \rightarrow \infty)$, so that (A.5) implies (2.3). This ends the proof of the implication " $(2.4) \Rightarrow(2.3) "$.

\section{References}

[1] Aldous, D.J. and Bandyopadhyay, A.(2005). A survey of max-type recursive distributional equations. Ann. Appl. Probab., 15(2), 1047-1110.

[2] Alsmeyer, G. and Iksanov, A.(2009). A log-type moment result for perpetuities and its application to martingales in supercritical branching random walks. Electron. J.Prob., 14, 289-313.

[3] Alsmeyer, G. and Kuhlbusch, D.(2010). Double martingale structure and existence of $\phi$-moments for weighted branching processes. Münster J. of Math., 3, 163-211.

[4] Barral, J.(1999). Moments, continuité, et analyse multifractale des martingales de Mandelbrot. Probab. Theory Relat. Fields, 113, 535-569. 
[5] Barral, J. and Jin, X. (2014). On exact scaling log-infinitely divisible cascades. Probab. Theory Related Fields, 160, 521-565.

[6] Biggins, J.D.(1977). Martingale convergence in the branching random walk. J. Appl. Probab., 14, 25-37.

[7] Biggins, J.D. and Kyprianou, A.E.(1997). Seneta-Heyde norming in the branching random walk. Ann. Probab., 25, 337-360.

[8] Biggins, J.D. and Kyprianou, A.E. (2005). Fixed points of the smoothing transform: the boundary case. Electron. J. Prob., 10(17), 609-631.

[9] Bingham, N.H. and Doney, R.A.(1974). Asymptotic properties of supercritical branching processes I: The Galton-Watson processes. Adv. Appl. Prob., 6, 711-731.

[10] Bingham, N.H. and Doney, R.A.(1975). Asymptotic properties of supercritical branching processes II: Crump-Mode and Jirina processes. Adv. Appl. Prob., 7, 66-82.

[11] Bingham, N.H., Goldie, C.M. and Teugels, J.L.(1987). Regular Variation. Cambridge Univ. Press, Cambridge.

[12] Buraczewski, D.(2009). On tails of fixed points of the smoothing transform in the boundary case. Stoch. Proc. Appl., 119(11), 3955-3961.

[13] Buraczewski, D. and Kolesko, K. (2014). Linear stochastic equations in the critical case. J. Difference Equ. Appl., 20, 188-209.

[14] Chen, X. (2015). A necessary and sufficient condition for the nontrivial limit of the derivative martingale in a branching random walk. Adv. Appl. Probab., 47, 741-760.

[15] de Haan, L.(1976). An Abel-Tauber theorem for Laplace transforms. J. London Math. Soc., 17, 102106.

[16] de Meyer, A. and Teugels, J.L.(1980). On the asymptotic behavior of the distributions of the busy period and service time in $M / G / 1$. J. Appl. Prob., 17, 802-813.

[17] de Meyer, A.(1982). On a theorem of Bingham and Doney. J. Appl. Prob., 19, 217-220.

[18] Durrett, R. and Liggett, T.(1983). Fixed points of the smoothing transformation. Z. Wahrsch. verw. Gebeite, 64, 275-301.

[19] Feller, W.(1971). An Introduction to Probability Theory and its Applications, Vol. II, 2nd ed. Wiley, New York.

[20] Guivarc'h, Y.(1990). Sur une extension de la notion de loi semi-stable. Ann. IHP, 26, 261-185.

[21] Hu, Y. and Shi, Z. (2009). Minimal position and critical martingale convergence in branching random walks, and directed polymers on disordered trees. Ann. Probab., 37, 742-789.

[22] Iksanov, A., Kolesko, K. and Meiners, M.(2019). Stable-like fluctuations of Biggins martingales. Stoch. Proc. Appl., to appear. Preprint is available at arxiv.org/pdf/1709.07362.

[23] Iksanov, A. and Polotskiy, S.(2006). Regular variation in the branching random walk. Theory of stochastic processes, $\mathbf{1 2}(\mathbf{2 8}), 38-54$.

[24] Kahane, J.P. and Peyrière, J.(1976). Sur certaines martingales de Benoit Mandelbrot. Adv. Math., 22, 131-145.

[25] Liang, X. and Liu, Q. (2011). Tail behavior of laws stable by random weighted mean. C. R. Math. Acad. Sci. Paris, 349, 347-352.

[26] Liang, X. and Liu, Q. (2015). Weighted moments for Mandelbrot's martingales. Electron. Commun. Probab., 20, 1-12. 
[27] Liu, Q.(1997). Sur une équation fonctionelle et ses applications: une extension du théorème de KestenStigum concernant des processus de branchement. Adv. Appl. Probab., 29, 353-373.

[28] Liu, Q.(1998). Fixed points of a generalized smoothing transform and applications to the branching random walk. Adv. Appl. Prob., 30, 85-112.

[29] Liu, Q.(2000). On generalized multiplicative cascades. Stoch. Proc. Appl., 86, 263-286.

[30] Lyons, R.(1997). A simple path to Biggins' martingale convergence for branching random walk. In: Athreya, K.B., Jagers, P. (Eds.), Classical and modern branching processes, IMA Volumes in Math. and its Appl., 84, Springer, Berlin, 217-221.

[31] Mikosch, T. and Samorodnitsky, G.(2000). The supremum of a negative drift random walk with dependent heavy-tailed steps. Ann. Appl. Probab., 10, 1025-1064.

[32] Rösler, U.(1992). A fixed point theorem for distributions. Stoch. Proc. Appl., 42, 195-214.

[33] Rösler, U., Topchii, V. and Vatutin, V.(2002). Convergence rate for stable weighted branching processes. In: Mathematics and Computer Science II (Versailles, 2002), Birkhäuser, Basel, 441-453.

[34] Rösler, U., Topchii, V. and Vatutin, V.(2003). The rate of convergence for weighted branching processes. Siberian Adv. Math., 12, 57-82.

[35] Shi, Z. (2015). Branching random walks. Lecture Notes in Mathematics, 2151. École d'été de Probabilités de Saint-Flour. Springer, Berlin, 133 pp.

[36] Volkovich, Y. and Litvak,N. (2010). Asymptotic analysis for personalized web search. Adv. Appl. Probab., 42, 577-604. 